\title{
Triboelectric nanogenerators: Fundamental physics and potential applications
}

\author{
Linglin ZHOU ${ }^{1,2}$, Di LIU $^{1,2}$, Jie WANG ${ }^{1,2,}$, Zhong Lin WANG ${ }^{1,3, *}$ \\ ${ }^{1}$ Beijing Institute of Nanoenergy and Nanosystems, Chinese Academy of Sciences, Beijing 100083, China \\ ${ }^{2}$ College of Nanoscience and Technology, University of Chinese Academy of Sciences, Beijing 100049, China \\ ${ }^{3}$ School of Materials Science and Engineering, Georgia Institute of Technology, Atlanta, GA 30332, USA \\ Received: 24 February 2020 / Revised: 26 March 2020 / Accepted: 27 March 2020 \\ (C) The author(s) 2020 .
}

\begin{abstract}
Based on the conjunction of contact electrification and electrostatic induction, triboelectric nanogenerators (TENGs) can harvest mechanical energy dispersed in our environment. With the characteristics of simple structure, light weight, broad material availability, low cost, and high efficiency even at low operation frequency, TENG can serve as a promising alternative strategy for meeting the needs of distributed energy for the internet of things and network. The major potential applications of TENG can be summarized as four fields containing micro/nano power sources, self-powered sensors, large-scale blue energy, and direct high-voltage power sources. In this paper, the fundamental physics, output performance enhancement, and applications of TENGs are reviewed to timely summarize the development of TENGs and provide a guideline for future research.
\end{abstract}

Keywords: contact electrification; self-powered system; energy harvesting; triboelectric nanogenerator

\section{Introduction}

As a ubiquitous phenomenon, the contact electrification (CE) has been known for 2,000 years. CE results from physical contact between two materials, along with the process of tribology and interfacial charge transfer, which is one of the most fundamental effects of electricity generation [1]. However, there is few applications for CE in modern industry, and is usually acted as a negative effect to be avoided because the introduced electrostatic charges can cause ignition, dust explosions, increased friction, energy losses, etc. [2]. The situation has changed until the triboelectric nanogenerators (TENGs) were invented as a new type of energy harvesting technology [3]. By a conjunction of triboelectrification (TE) and electrostatic induction, TENGs can transform a variety of mechanical energy in our daily life to electricity. As for this electric power generation technology, TE generates static polarized charges on the material surface through physical contact between two materials, while electrostatic induction makes the conversion of mechanical energy into electric power by mechanical separation generating the change of electrical potential.

Since the first report in 2012, TENGs have been developed very rapidly and attracted extensive attention on a global scale [4-8]. Based on large efforts including basic principles and modes proposing, material improvement, device design, structural optimization, and surface modification, the area power density and conversion efficiency of TENGs have reached to $500 \mathrm{~W} \cdot \mathrm{m}^{-2}$ and $>50 \%$, respectively [9]. With many desirable merits of simple structure, light weight, broad material availability, low cost, and high efficiency even at low operation frequency, TENGs can be used to harvest various mechanical energy that is ubiquitous but wasted in our daily life [10-12], and thus has been proven to be a promising alternatively method for

* Corresponding authors: Jie WANG, E-mail: wangjie@binn.cas.cn; Zhong Lin WANG, E-mail: zhong.wang@mse.gatech.edu 
satisfying the demands of distributed energy for the internet of things (IoTs) and network [13]. In addition, TENG has demonstrated its potential application including powering small and mobile electronic devices as micro/nano power sources, coupling with existing sensor device for establishing self-powered system, and applying as high-voltage (HV) power source for HV applications [14-19]. Moreover, networks of TENG units can be effectively applied for harvesting ocean waves with low frequency, with the prospect of worldwide energy production at large scale [20-24].

With the rapid development of TENG, we prepare this paper to timely summarize the development of TENGs and provide a guideline for future research. In this review, we will first focus on the fundamental comprehension of TENG, containing the origin of TE and its mechanism, following by an introduction of the basic operating modes. Then, performanceenhancement methodology for TENG will be elaborated, including enhancement of surface charge density, improvement of output power, and power management. Furthermore, this paper will give a summary about the recent important progresses in TENG applications. Finally, based on the road map of TENG research with identify prioritized directions, some new horizons are presented for exploring the study and application of TENG.

\section{Fundamental physics of TENGs}

\subsection{Origin of triboelectrification}

Aiming to build the scientific basis of TENG and enhance its performance, fundamental mechanism of TE must be studied. Although this effect has been known for two thousand years, the corresponding fundamental science is blurry until a fundamental understanding was provided in 2019. By anatomizing the corresponding studies about TE in nano-scale, a conclusion has been obtained that electron transfer is the dominant, if not exclusive, mechanism for TE [1]. For understanding the origin of TE, Wang and Wang [1] proposed the interatomic interaction model to explain TE on atomic level, where TE occurs between two atoms with partial overlap in their electronic clouds or wave functions. (i) represents the general form of interatomic interactions in Fig. 1(a). If a bond is formed between two atoms, it is meaning that partial overlap occurs in electronic clouds or wave functions, where an equilibrium state with interatomic distance of $a$ is built. When an external force, one of the most determining factors in TE, is applied to produce physical contact between the two surfaces, a local high pressure will be generated at certain points even at atoms, resulting a shorter interatomic distance $x$ than $a$ between the contacting points. In this case, a repulsive force will be generated between the two atoms with the enhancement of overlap in electronic clouds or wave functions, and therefore electrons transfer from one surface to the other one, resulting in TE between the two surfaces ((ii) in Fig. 1(a)). On the contrary, the two atoms have a tendency to attract each other because of the lower overlap of electron clouds, which may break their bond ((iii) in Fig. 1(a)).

Recently, the interatomic interaction model was confirmed by using tapping mode atomic force microcopy (AFM) to control the degree of electro-cloud overlap between two atoms [25]. The tapping mode AFM and the illustration of attractive region and repulsive region on the sample surface are displayed in Fig. 1(b). The tip is excited by the piezoelectric ceramic to vibrate at certain amplitude before approaching to the sample surface. If a force is applied on the vibrating tip, the amplitude of the tip will decrease and the vibration phase will shift due to interference on its harmonic oscillating behavior. The shift direction of the phase depends on the direction of the applied force. Specifically, the tip-sample interaction force is dependent on the set amplitude when the free amplitude is constant, while the phase shift of the vibrating tip changes with the set amplitude, and interaction regions of the tip-sample interaction force can be reflected by the direction of the phase shift. The shift of the tip when it taps on the $\mathrm{Si}_{3} \mathrm{~N}_{4}$ surface at different set amplitudes is displayed in Figs. 1(c)-1(e). When the free amplitude was $50 \mathrm{~nm}$, the phase shift was always negative, which means the tip cannot enter the repulsive region. When the free amplitude increased to 70 and $100 \mathrm{~nm}$, the phase shift of the tip will reverse to be positive if the set amplitude is smaller than a threshold value. In these parameters, the tip worked in the attractive region when the free amplitude is $50 \mathrm{~nm}$ (Fig. 1(c)) and in the repulsive region when the free amplitudes are 70 and $100 \mathrm{~nm}$ (Figs. 1(d) and 1(e)). 


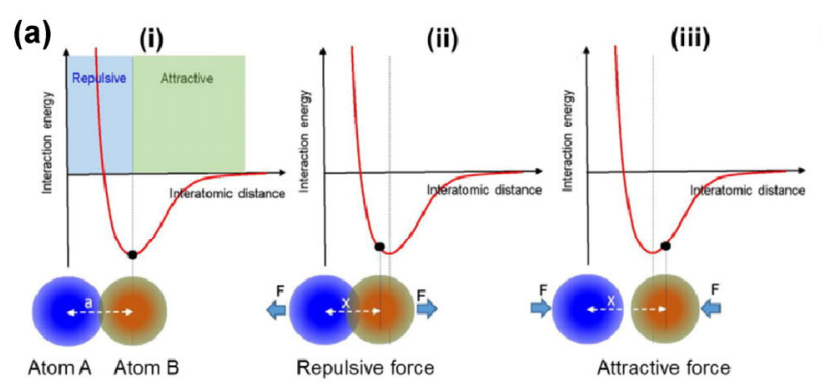

(b)
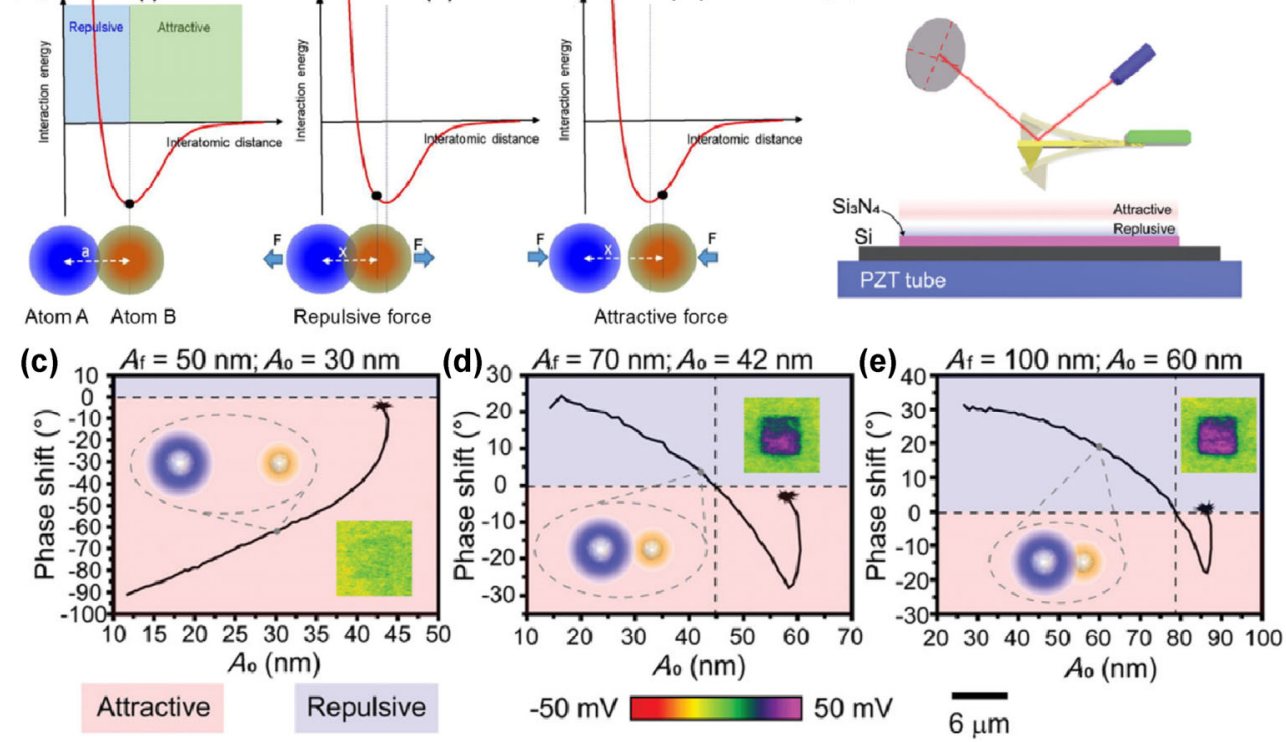

Fig. 1 Charge transfer model of triboelectrification. (a) Interatomic interaction model with the relationship of interaction energy curve and the interaction of the atoms. Reproduced with permission from Ref. [1]. ( E Elsevier Ltd., 2019. (b) Tapping mode AFM and the illustration of attractive region and repulsive region on the sample surface. $(\mathrm{c}-\mathrm{e})$ The phase shift at different set tapping amplitudes and the charge transfer between tip and $\mathrm{Si}_{3} \mathrm{~N}_{4}$ sample with different free amplitude $\left(A_{\mathrm{f}}\right)$ and set tapping amplitude $\left(A_{0}\right)$. Reproduced with permission from Ref. [25]. (C) WILEY-VCH Verlag GmbH \& Co. KGaA, 2020.

These results show that the $\mathrm{Si}_{3} \mathrm{~N}_{4}$ surface obtained positive charges when the tip tapping in the repulsive region on the $\mathrm{Si}_{3} \mathrm{~N}_{4}$ surface, while the charges transfer between the tip and $\mathrm{Si}_{3} \mathrm{~N}_{4}$ did not occur when the tip tapping in the attractive region (Fig. 1(c)). These results suggest that the electron transfer between tip and sample occurs only if the two surfaces interact in the repulsive-force region, which is consistent with the interatomic interaction model.

Besides electron transfer, the TE also involves the mechanisms of chemical changes and material transfer between the contacting surfaces. Baytekin et al. [26] studied the chemical changes on a pair of polymers by using X-ray photoelectron spectroscopy (XPS) and the feature distinct elemental peaks confirmed the presence of material transfer. Once PTFE and polydimethylsiloxane (PDMS) upon contact, the F 1s signal in the XPS spectrum at $690 \mathrm{eV}$ presents in the spectrum of PDMS, while Si $2 \mathrm{p}$ at $102.5 \mathrm{eV}$ and $\mathrm{O} 1 \mathrm{~s}$ at $533 \mathrm{eV}$ peaks present in the spectrum of PTFE. Moreover, the increased intensities of above-mentioned peaks also obtained with increasing the cycles of PTFEPDMS contacts. These measurements indicate that $\mathrm{TE}$ is a complex process containing a combination of bond cleavage, chemical changes, and material transfer taking place within distinct patches of nanoscopic dimensions.

The surface state model can be used to further explain the mechanism of TE between two dielectrics [27]. Assuming the existence of surface and defect states in the bandgap owing to the presence of surface and possibly point defects, the electric structure of dielectric surface can be represented by a band diagram with conduction band (CB) and valence band (VB) $[1,28]$. The difference in electron structures between two materials, resulting in the energy of unoccupied surface states of dielectric lower than that of occupied surface states in dielectric B (Fig. 2(a)). Once the two dielectrics contact with each other, TE will occur by the electrons transfer from the surface of dielectric A to the surface of dielectric B (Fig. 2(b)). Even after separation, these transferred electrons in dielectric $B$ will not return to the surface of dielectric $A$, leading to net negative charges on dielectric $B$ and net positive charges on dielectric A (Fig. 2(c)).

\subsection{Maxwell current}

From the fundamental physics, the theory of TENG 
(a)

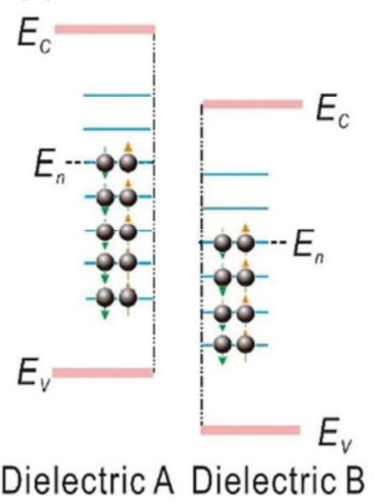

(b)

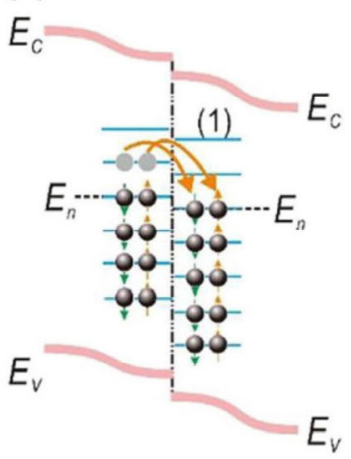

(c)

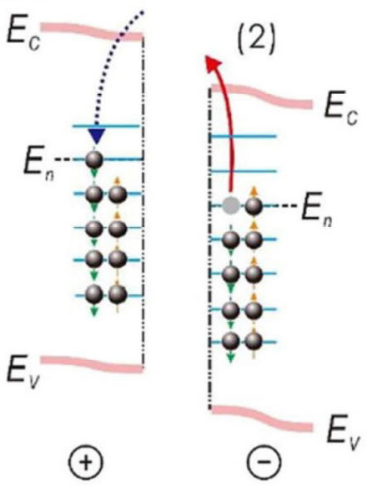

Fig. 2 Surface state model to explain the mechanism of TE between two dielectrics. Surface state model with charge transfer: (a) before contact, (b) in contact, and (c) after contact between two different dielectric materials for a case that $E_{n}$ of the dielectric A is higher than that of the dielectric B. Reproduced with permission from Ref. [1]. C) Elsevier Ltd., 2019.

is origin from the Maxwell's displacement $[29,30]$ defined as

$$
J_{D}=\frac{\partial D}{\partial t}+\frac{\partial P_{s}}{\partial t}=\varepsilon \frac{\partial E}{\partial t}+\frac{\partial P_{s}}{\partial t}
$$

where $J_{D}$ is the total displacement current density, $D$ is the electric displacement field, $P_{s}$ is the polarization field created by the electrostatic surface charges resulting from triboelectric effect, $E$ is the electric field, and $\varepsilon_{0}$ is the vacuum permittivity. The first term $\varepsilon(\partial E / \partial t)$ is the induced current by the varying electric field which gives the birth of electromagnetic waves, while the second term $\partial P_{s} / \partial t$ is the current attributed to the surface polarization which is the origin of nanogenerators, and is simply referred as Wang term. Thus, the displacement current $\left(I_{D}\right)$ is a surface integral of $J_{D}$ :

$$
I_{D}=\int J_{D} \mathrm{~d} s=\int \frac{\partial D}{\partial t} \mathrm{~d} s=\frac{\partial}{\partial t} \int \nabla \cdot D \mathrm{~d} r=\frac{\partial}{\partial t} \int \rho \mathrm{d} r=\frac{\partial Q}{\partial t}
$$

where $Q$ is the total free charges on the electrode, $\rho$ is the distribution of free charges in space. From Eq. (2), the internal circuit in TENG is dominated by the displacement current, and the observed current in the external circuit is the capacitive conduction current. The internal circuit and external circuit can meet at the two electrodes, forming a complete loop. Therefore, the displacement current is the intrinsic physical core of current generation and it is the internal driving force, and the capacitive conduction current in an external circuit is the external manifestation of displacement current [30].

The basic structure of contact-separation mode is used to illustrate this theory (Fig. 3), which consists of two electrodes connected to different dielectric films, with dielectric permittivity of $\varepsilon_{1}$ and $\varepsilon_{2}$ and thicknesses of $d_{1}$ and $d_{2}$, respectively. The surface charge density induced by $\mathrm{CE}$ is $\sigma_{T}(t)$, which finally reaches a saturation after the initial contacts between the two films, and is independent of the gap distance $z$. Driven by the electrostatic filed, the density of free electrons accumulated on the surface of electrode is $\sigma(z, t)$, which is a function of $z(t)$. In the two dielectric films, the electric fields are $E z=\sigma(z, t) / \varepsilon_{1}$ and $E z=-\sigma(z, t) / \varepsilon_{2}$, respectively. In the gap, the electric field is $E z=$ $-\left[\sigma(z, t)-\sigma_{T}\right] / \varepsilon_{0}$. Between the two electrodes, the

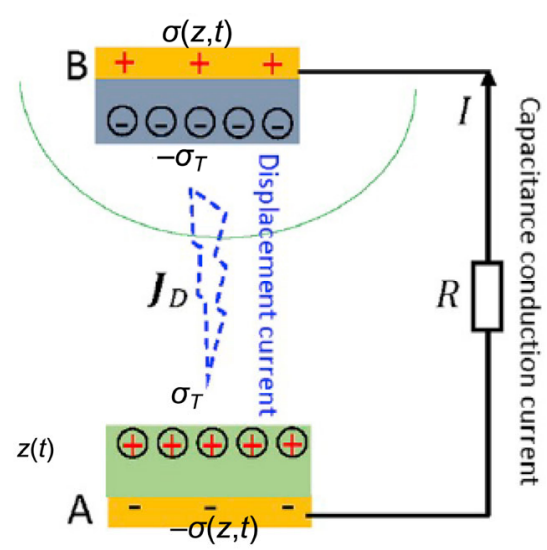

Fig. 3 Working mechanisms of TENG with contact-separation mode. Reproduced with permission from Ref. [30]. (C) Elsevier Ltd., 2020. 
relative potential drop can be calculated as

$$
V=\left\{-\sigma(z, t)\left(d_{1} / \varepsilon_{1}+d_{2} / \varepsilon_{2}\right)-z\left[\sigma(z, t)-\sigma_{T}\right]\right\} / \varepsilon_{0}
$$

Under short-circuit condition, $V=0$ :

$$
\sigma(z, t)=z \sigma_{T} /\left(d_{1} \varepsilon_{0} / \varepsilon_{1}+d_{2} \varepsilon_{0} / \varepsilon_{2}+z\right)
$$

Therefore, the corresponding displacement current density is

$$
\begin{aligned}
J_{D}= & \frac{\partial D_{z}}{\partial t}=\frac{\partial \sigma(z, t)}{\partial t}=\sigma_{T} \frac{\mathrm{d} z}{\mathrm{~d} t}\left(\frac{d_{1} \varepsilon_{0}}{\varepsilon_{1}}+\frac{d_{2} \varepsilon_{0}}{\varepsilon_{2}}\right) \\
& /\left(d_{1} \varepsilon_{0} / \varepsilon_{1}+d_{2} \varepsilon_{0} / \varepsilon_{2}+z\right)^{2}+\frac{\mathrm{d} \sigma_{T}}{\mathrm{~d} t} z \\
& /\left(d_{1} \varepsilon_{0} / \varepsilon_{1}+d_{2} \varepsilon_{0} / \varepsilon_{2}+z\right)
\end{aligned}
$$

From Eq. (5), the output characteristics of the TENG can be illustrated that the displacement current density is proportional to the speed at which the two dielectric films are being separated or contacted, and is also in proportion to surface charge density. Considering the existence of an external load $R$, the current transport equation for TENG can be given by

$$
\begin{array}{rl}
R & A \frac{\partial \sigma(z, t)}{\partial t} \\
& =\left\{-\frac{z \sigma\left[\sigma(z, t)-\sigma_{T}\right]}{\varepsilon_{0}}-\sigma(z, t)\left(\frac{d_{1}}{\varepsilon_{1}}+\frac{d_{2}}{\varepsilon_{2}}\right)\right\} / \varepsilon_{0}
\end{array}
$$

where $A$ is the area of the electrode. From the mechanism of the basic model of the TENG, we can find that the theoretical origin of the TENG is Maxwell's displacement current.

\subsection{Four modes of TENG}

Since the first report of TENG in 2012, four different modes have been invented according to motion manner of the triboelectric layer and electrode arrangement, which are vertical contact-separation mode, lateralsliding mode, single-electrode mode, and freestanding triboelectric-layer mode, respectively (Fig. 4). The detailed theoretical models of the mentioned modes have been widely elaborated in previous work, therefore a concise description is given in this paper as follows.

As the most basic and common structure, the vertical contact-separation mode [31, 32] consists of a top electrode and a back electrode connected to dielectric (a)

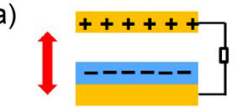

Contact-separation mode

(c)

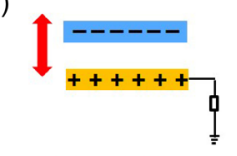

Single-electrode mode Freestanding triboelectric-layer mode (b)

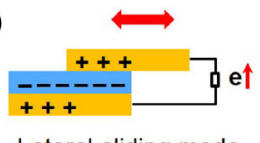

(d)

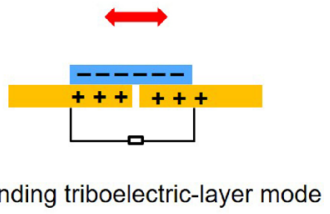

Fig. 4 Four fundamental modes of TENG with (a) vertical contactseparation mode, (b) sliding mode, (c) single-electrode mode, and (d) freestanding triboelectric-layer mode.

film. Driving by applied force, these two parts move with each other in the vertical direction to the interface, resulting in the generation of charges. During contacting or separating process, the potential changes between electrodes, and thus external current flow is produced with an external circuit connected.

The lateral-sliding mode [33, 34] has the same structure as that of vertical contact-separation mode, which is driven by the force that is parallel to the interface, resulting in the relative displacement between the material surface and thus dense triboelectric charges. With the periodic change of the contact surface area, the potential difference changes between the electrodes, and then the external current flow is generated to balance the potential difference. During the moving process, the two surfaces contact with each other without any air gap distance in principle, a compact package can be achieved in this mode by using rotation-induced sliding.

The above-mentioned working modes of TENG demand the moving object with attaching an electrode. Considering most of the motion is free in actual scenario, the single-electrode mode $[35,36]$ was invented, where the object can move freely without connecting an electric conductor. In this mode, only one electrode needs to connect with the ground, serving as the reference electrode. The charges will generate on the interface after contacting with freely moving object, and the potential distribution induced by charged surface changes during the motion process, causing the external current flow.

As for the freestanding triboelectric-layer mode [37], it only needs a freely motion part. This mode contains a dielectric layer and a pair of symmetric electrodes, 
the asymmetric potential distribution is produced with the freely motion part changing its position, following by the electrons moving between the two electrodes, and finally resulting in the electrical output.

\section{Enhancing performance of TENGs}

\subsection{Enhancing the surface charge density}

Considering four working modes of TENG with different mechanical triggering and broad material availability, a common standard of figure-of-merit was proposed for quantifying performance of TENGs from the view of structure and material [38]. From the built-up voltage $(V)$-total transferred charges $(Q)$ curve, the maximized energy output can be achieved by using the TENG operation cycle. Based on this cycle, a performance figure-of-merit (FOMp), composed of a structural FOM $\left(\mathrm{FOM}_{\mathrm{S}}\right)$ and a material FOM $\left(\mathrm{FOM}_{\mathrm{m}}\right)$, was proposed for evaluating the performance of TENGs. Besides, the $\mathrm{FOM}_{\mathrm{m}}$ can be regarded as the square of the surface charge density, which is related to the characteristic of the materials. As a dominate parameter for the performance of TENGs, the power density is quadratically related to the surface charge density, which has been enhanced via numerous research efforts until now. There are mainly four strategies employed to achieve a high charge density including selecting materials, enhancing the contact intimacy, optimizing the working environment, and utilizing a thin film as dielectric layer. The first strategy to improve the charge density of TENG is choosing a pair of materials who can create large static electricity by rubbing with each other [39-43]. For better selecting materials, a standard method that can precisely quantify the triboelectric charge density of a general material is highly required. Based on these, a universal standard method was proposed to quantify the triboelectric series for a wide range of polymers, and thus establish quantitative triboelectrification as a fundamental materials property [44]. For maximizing the contact intimacy between two materials, a liquid metal of mercury was employed as the reference material to ensure shape adaptability and softness. By measuring over 50 materials, the results were summarized to build a triboelectric order as shown in Fig. 5. The normalized triboelectric charge density represents the characteristic of the materials to obtain or loss electrons after TE. Therefore, an appropriate material can be selected by using the standards evaluation method or accounting to quantified triboelectric series to enhance the triboelectric charge density.

The second strategy to improve the triboelectric charge density is based on enhancement of contact intimacy. One straightforward approach is to fabricate micro/nanostructures onto the triboelectric materials, such as polypyrrole (PPy) with horn-like morphology (Fig. 6(a)) [45]. The PPy-based TENG consists of a PTFE as a triboelectric layer and hollow hornlike PPy (hPPy) as the other triboelectric electrode. Compared with the triboelectric charge density generated by TENG using general PPy film (cauliflower-like PPy, cPPy) and copper film as triboelectric electrode, the charge density of hPPy-based TENG reached up to $84 \mu \mathrm{C} \cdot \mathrm{m}^{-2}$, which is much higher than those of cPPy or Cu-based TENG with about $40 \mu \mathrm{C} \cdot \mathrm{m}^{-2}$. The enhancement of charge density can be contributed to the larger contact area derived from the micro/nanostructure of triboelectric material and more efficient triboelectrification caused by a high contact intimacy from the micro/nanostructure of hPPy with more shape-adaptive. Owing to soft materials and fragmental contacting structure, a larger contact area can be realized in atomic dimensions, which finally improves the contact intimacy of the surfaces. Therefore, soft materials and fragmental contacting structure tend to improve the contact effectiveness of the surface under the same driven force, and thus a higher triboelectric charge density for TENG can be attainted. Wang et al. [46] proposed a tube-like TENG with silicone-rubber-based material as triboelectric layer and electrode (Fig. 6(b)), which achieved a high surface charge density of $250 \mu \mathrm{C} \cdot \mathrm{m}^{-2}$. The tube-like TENG mainly consists of two parts, one is a dielectric layer attached to an outer electrode to form a tube, and the other is a belt-like inner electrode adhered to the interior surface of the tube and shape into a helix extending along the inside of the tube. Silicone rubber and the mixture of silicone rubber, carbon black and carbon nanotubes were used as dielectric layer and electrode, respectively, which endows a high intimacy for the tube-like TENG. With 


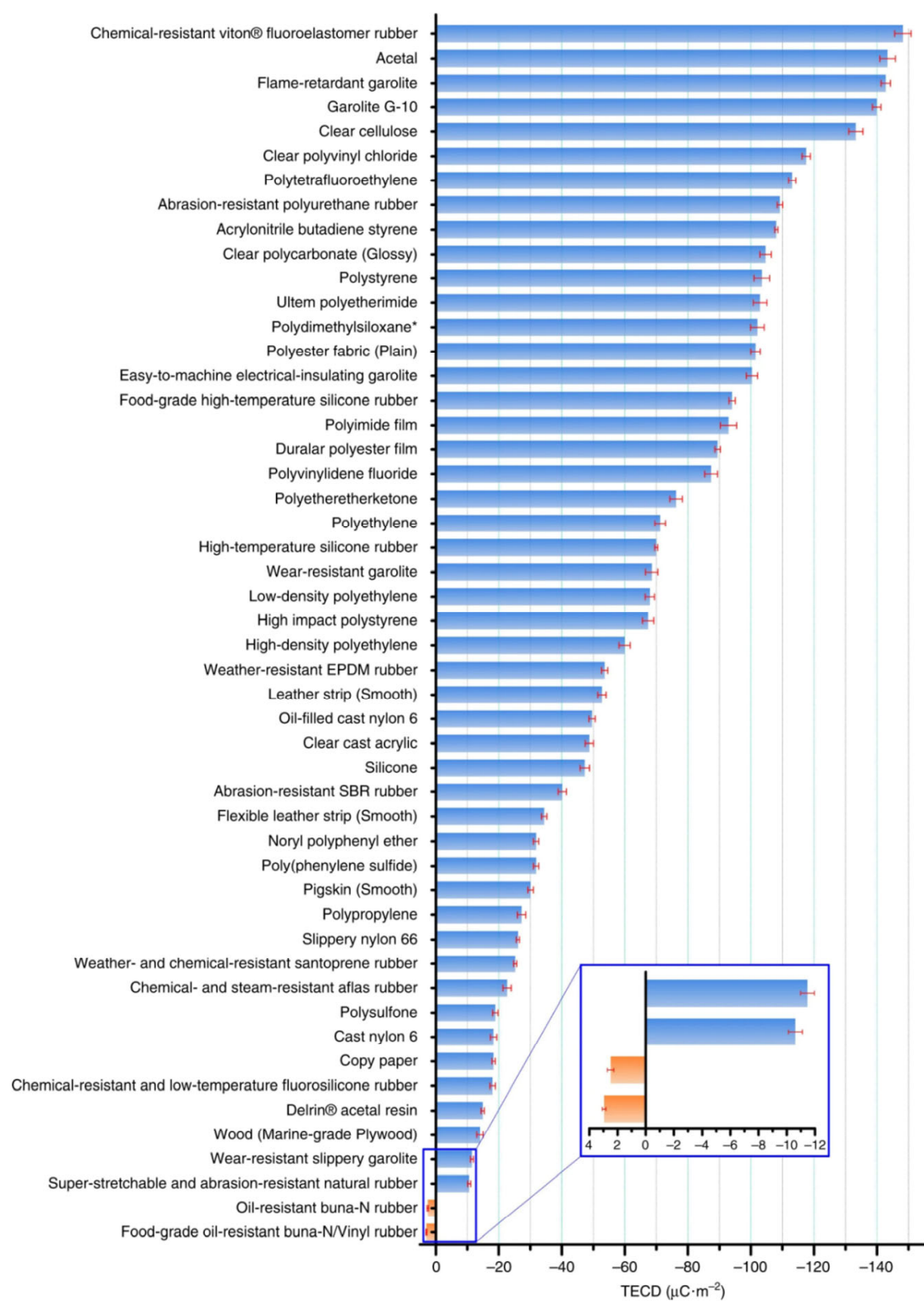

Fig. 5 Quantified triboelectric series. Reproduced with permission from Ref. [44]. (C) Springer Nature, 2019.

only the energy harvested from walking or jogging, a stable performance for the helix-belt structured TENG can be achieved by various directions triggering, which can immediately and continuously drive many wearable electronics including electronic watch and fitness tracker.

The third method is to optimize the working environment such as atmosphere and air pressure. Lin et al. [47] investigated atmosphere effects on the performance of TENG at nano-scale by using atomic force microscopy and Kelvin probe force microscopy. In different atmosphere conditions including air, $\mathrm{O}_{2}$,
$\mathrm{N}_{2}$, and $\mathrm{Ar}$, the contact electrification between $\mathrm{Pt} / \mathrm{Ir}$ coated tip and kinds of dielectric materials such as $\mathrm{SiO}_{2}$, AIN, polyvinyl chloride (PVA), and polymethyl methacrylate (PMMA) was studied, the results indicated that $\mathrm{O}_{2}$ molecules can shift the highest occupied surface state level of dielectrics and make the dielectrics tend to be negatively charged, therefore the output performance of TENG can be improved by changing the $\mathrm{O}_{2}$ concentration in the atmosphere (Fig. 7(a)). Considering the presence of air breakdown, the effect of air pressure on output performance of TENG has been well studied. By operating in vacuum 
(a) I
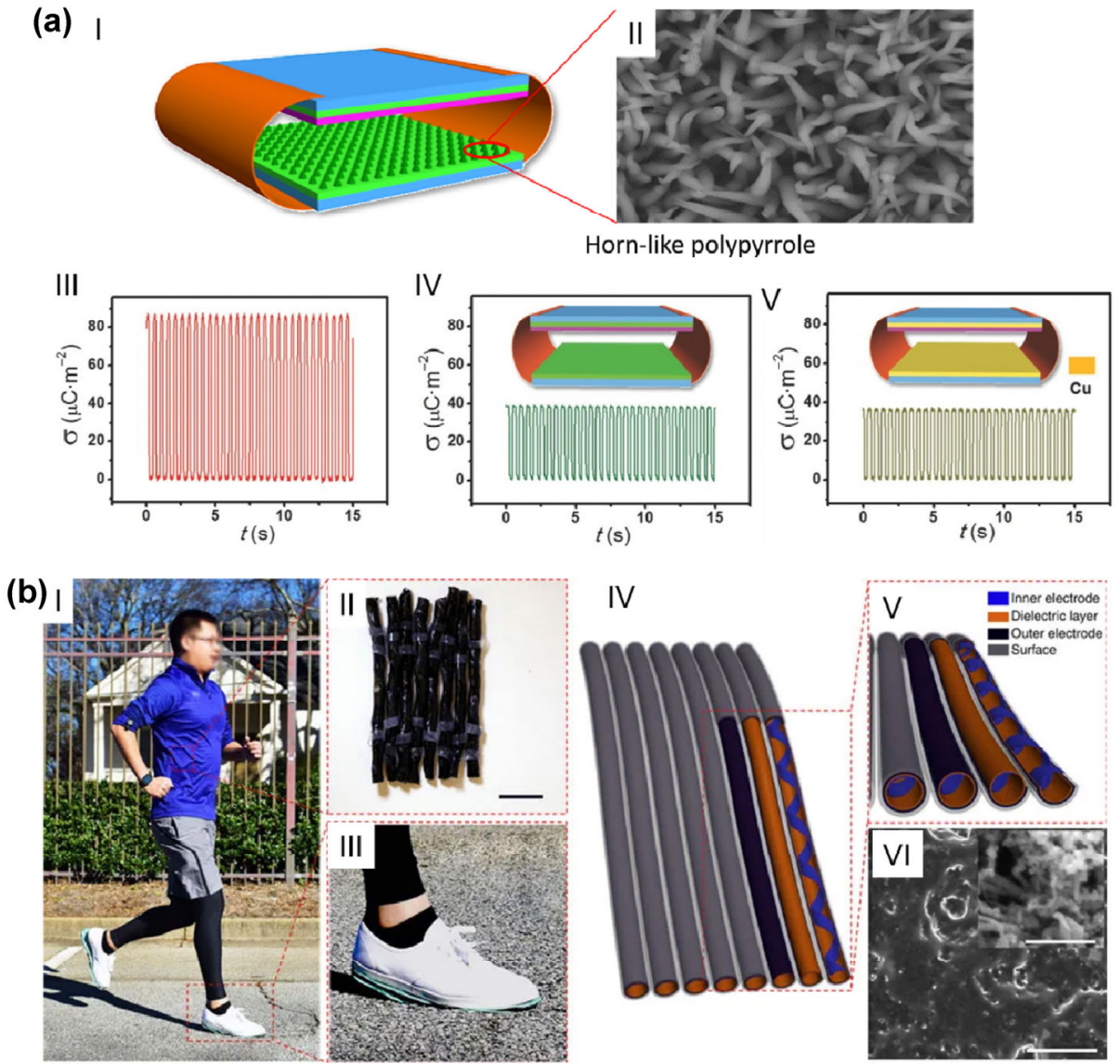

Fig. 6 Improvement of triboelectric charge density by enhancing contact intimacy. (a) PPy-based TENG: (I) schedule of the integrated self-charging power system from PPy-based TENG; (II) SEM image of hPPy with horn-like morphology; (III, V) triboelectric surface charge density of the TENG with hPPy, cPPy, and copper as triboelectric electrode, respectively. Reproduced with permission from Ref. [45]. (C) WILEY-VCH Verlag GmbH \& Co. KGaA, 2015. (b) Tube-like TENG powering wearable electronics such as (I) an electronic watch and a fitness tracker; (II) photograph of tube-like TENG; (III) photograph of tube-like TENG fixed under a shoe; (IV) structure sketch of the TENG tubes; (V) enlarged view of the TENG structure; (VI) SEM image of the triboelectric electrode surface. Reproduced with permission from Ref. [46]. C) Springer Nature, 2016.

( $P \approx 10^{-6}$ torr), the charge density of $\mathrm{Cu}$-PTFE-based TENG can be boosted to a record-high value of $660 \mu \mathrm{C} \cdot \mathrm{m}^{-2}$, where one of the biggest constraints, air breakdown, is avoided [48]. By introducing a ferroelectric material (BT ceramic) layer between the triboelectric PTFE layer and the back electrode, the charge density further improved to a higher record value of $1,003 \mu \mathrm{C} \cdot \mathrm{m}^{-2}$, which is the coupling effects of surface polarization from triboelectrification and hysteretic dielectric polarization from the ferroelectric material in vacuum (Fig. 7(b)). The attained value $\left(1,003 \mu \mathrm{C} \cdot \mathrm{m}^{-2}\right)$ is near to the limit of dielectric breakdown for PTFE material $\left(\sim 1,115 \mu \mathrm{C} \cdot \mathrm{m}^{-2}\right)$. Therefore, this work provided a new optimization methodology for TENG.
The promising method to achieve an ultrahigh surface charge density instead of operation in the high vacuum environment is by using a thin film as dielectric layer [48], while the high surface charge density is difficult to realize only through triboelectrification effect and electrostatic induction effect in atmosphere. By using thin film as dielectric layer, high surface charge densities in atmosphere can be realized by ions injection technology $[49,50]$, an external-charge TENG pumper [51, 52], and self-charge excitation TENG system [53], respectively. By injecting enough negative ions onto the Kapton film surface via an air-ionization-gun when the two electrodes of TENG were separated, a high surface charge density of $1,090 \mu \mathrm{C} \cdot \mathrm{m}^{-2}$ can be obtained in atmosphere with 
(a)
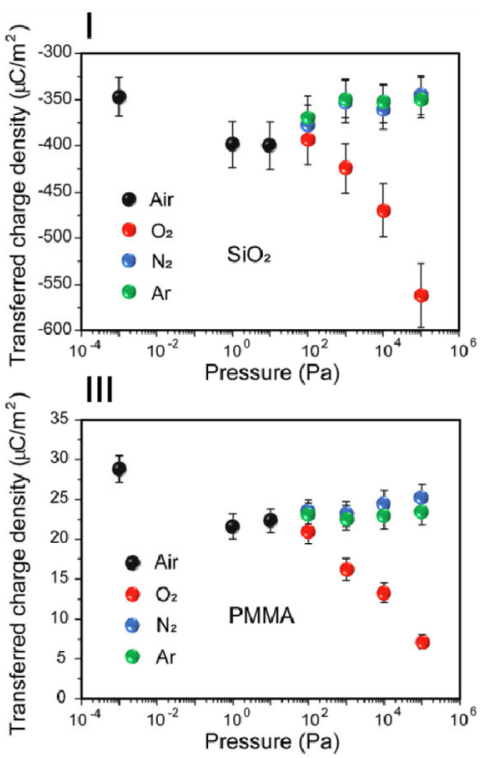
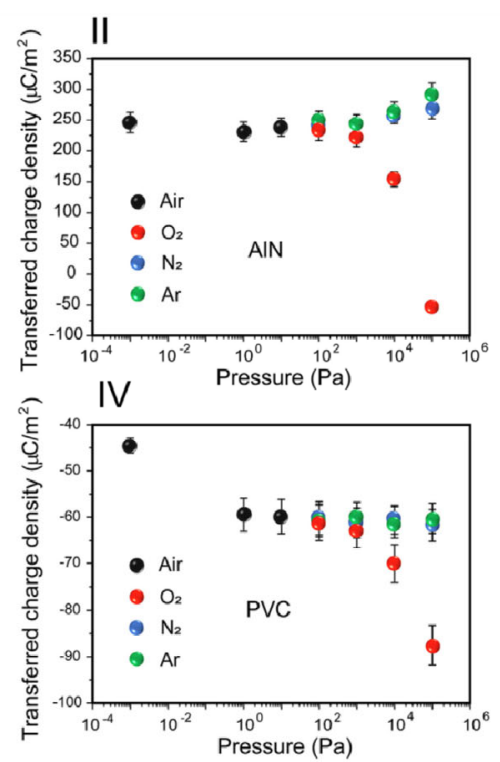

(b) 1

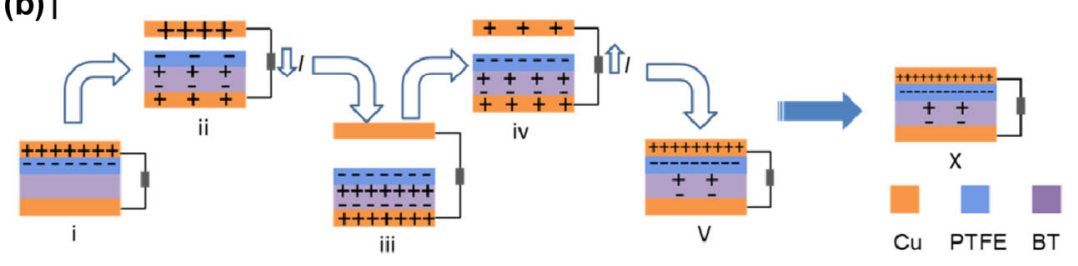

II

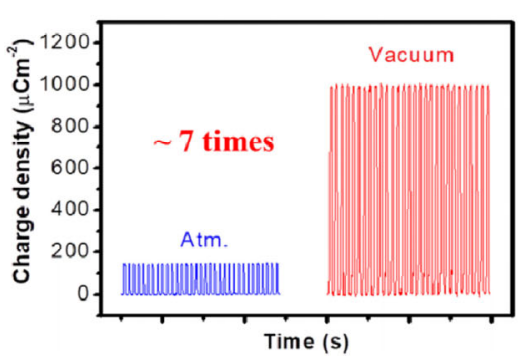

BT: Barium titanate

III

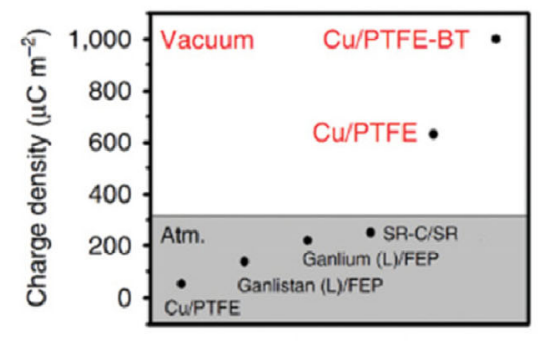

Fig. 7 Improvement of triboelectric charge density by optimizing working environment. (a) Tansferred charge density on the (I) $\mathrm{SiO}_{2}$, (II) AIN, (III) PMMA, and (IV) PVC surfaces induced by contacting with the tip in different atmospheres at different gas pressures. Reproduced with permission from Ref. [47]. ( Elsevier Ltd., 2019. (b) Working mechanism and output performance of TENG with the coupling effects of surface and dielectric polarization: (I) working mechanism of the fabricated TENG; (II) charge density of the TENG in atmosphere and high vacuum ( $P \approx 10^{-6}$ torr); (III) comparison of the output charges density measured in this work with previously reported ones. Reproduced with permission from Ref. [48]. () Springer Nature, 2017.

Kapton film of $6 \mu \mathrm{m}$ [49]. However, the obtained high charge density is unstable because of the lower charge accumulation and the faster decay process. External-charge TENG pumper proves a promising way to solve this problem, where the energy output from another TENG is utilized to improve the charge density. A typical work is the self-improving TENG (SI-TENG) proposed by Cheng et al. [51], which achieved the enhancement of the charge density and charge accumulation speed of TENG without air breakdown. The SI-TENG mainly consists of two parts: part I is a conventional contact-separation mode TENG with polyvinylidene fluoride (PVDF) and polyamide-6 (PA-6) film as the triboelectric materials, and part II is a plane parallel capacitor structure (PPCS) containing two electrodes covered with PVDF/ epoxy resin (EP) films. As shown in Fig. 8(a), these two parts are connected with each other by coupling 
(a)

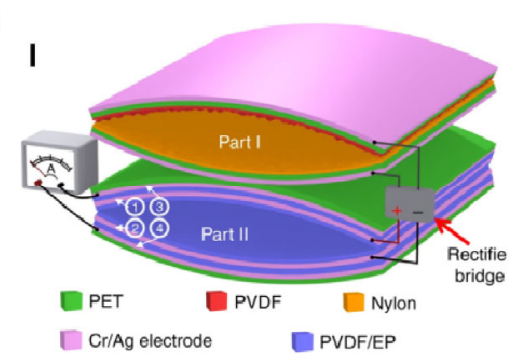

II

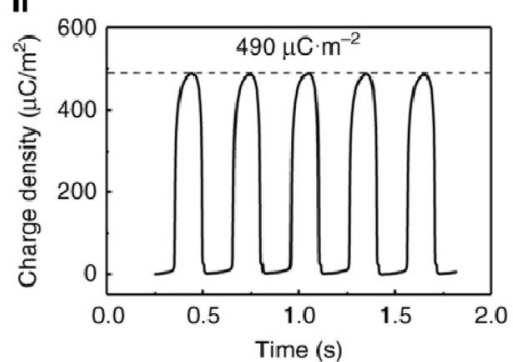

(b) 1
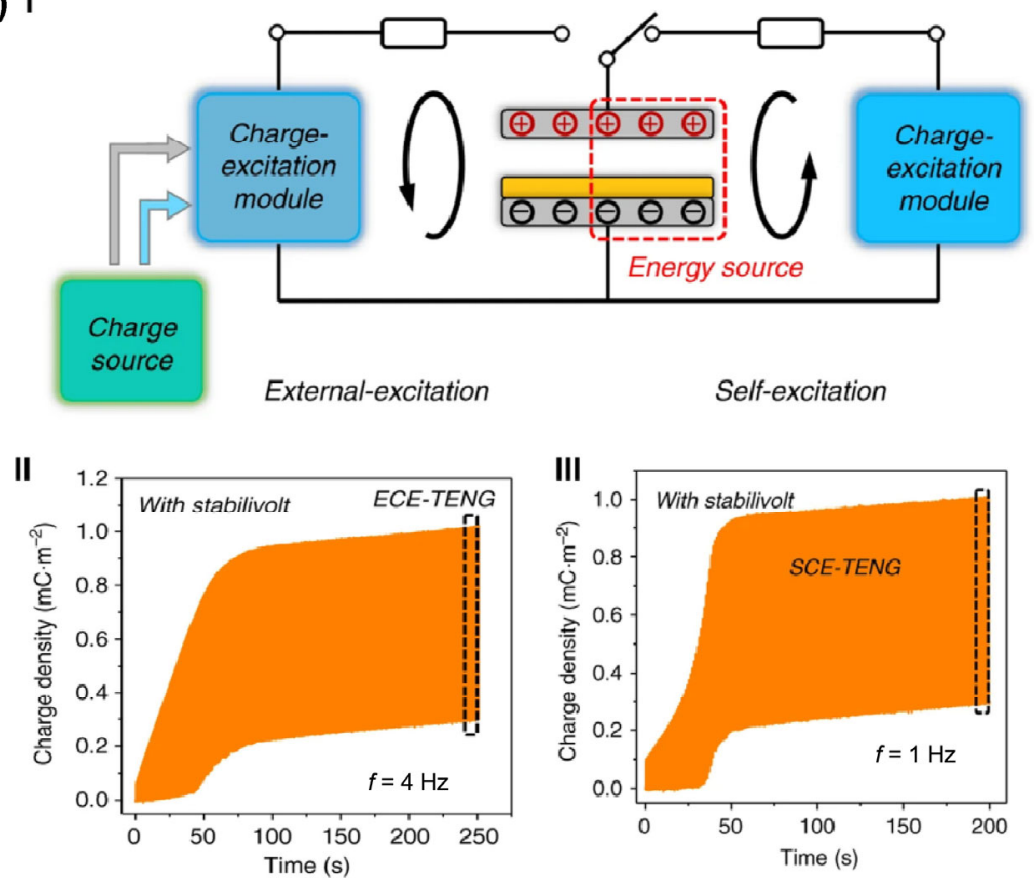

Fig. 8 Improvement of triboelectric charge density by using charge excitation methods. (a) Schematic diagram and transferred charge density of the SI-TENG. Reproduced with permission from Ref. [51]. (C) Springer Nature, 2018. (b) Working mechanism and output performance of both external and self-charge excitation TENG: (I) the fundamental scheme of both external and self-charge excitation TENG; (II, III) the charge density of ECE-TENG and SCE-TENG, respectively. Reproduced with permission from Ref. [53]. C Springer Nature, 2019.

the electrodes of part I with electron 1 and 2 of part II through a rectifier bridge. The AC current produced by part I can transfer to the DC signal, and then send to and finally stored in the PPCS, resulting in a high charge density in the device. By integrating with PPCS and contact-separation mode TENG, the SITENG reached a maximum effective charge density of $490 \mu \mathrm{C} \cdot \mathrm{m}^{-2}$ in an air environment. It's worth noting that effective charge density of the device is highly dependent on the voltage of part I, and a higher voltage of part I can effectively improve the performance of the SI-TENG. Moreover, the output performance of SI-TENG can be further enhanced by increasing the layer of part II in each device. Besides, by using the
SI-TENG, the charge accumulation speed is also improved, which is up to 5.8 times compared with an ordinary TENG. The second strategy is utilization part of the energy from the TENG itself to improving the charge density, which can largely improve the integration and charge accumulation efficiency of the device. Accounting to the mechanism of traditional magnetic excitation generators, a self-charge excitation TENG system was developed to attain a high and stable output, which was achieved by charge transferring between the TENG capacitor and external capacitors [53]. Different from the previous methods of exciting charge on the dielectric triboelectric layer or floating metal layer, the charges are directly transmitted 
to the electrodes of TENG in this work which can achieve both external and self-excitation (Fig. 8(b)). The system mainly contains the main TENG, output load, and the charge excitation system to transmit the charge to the electrode of TENG. For the external charge excitation TENG, it is composed of a basic excitation TENG, and the main TENG both with contact-separation mode, where thin Kapton film is employed as dielectric layer of the main TENG to realize a large capacitance variation, and buffer layers including flexible silicone, foam, and liquid cushion in the bottom were applied to ensure contact intimacy between the electrode and dielectric layer. Importantly, a voltage-multiplying circuit (VMC) and Zener diode were applied to a high and stable DC output for the main TENG. Furthermore, a self-charge excitation TENG system was designed by using a self-voltagemultiplying circuit derived from VMC. Based on the charge transfer between the main TENG and the external capacitor group, the basic power generation for the main TENG and output for external load can be achieved. Especially, utilizing the VMC and selfVMC with Zener diode as voltage stabilization element, a high excitation voltage was achieved to inject charges to the electrodes of the main TENG, meanwhile a stable output was obtained to avoid dielectric breakdown, and finally an effective charge output density of $1.25 \mathrm{mC} \cdot \mathrm{m}^{-2}$ was realized for both external charge excitation TENG and self-charge excitation TENG system in air environment with a $5 \mu \mathrm{m}$ Kapton film as dielectric layer.

\subsection{Enhancing the output power}

Aiming to meet the requirement of practical application, advanced structure designs have been explored to boost the output power. The milestone work of a radial-arrayed rotary TENG with micro-sized sectors on the surface was reported by Zhu et al. [54]. Using this structure, a high output power of $1.5 \mathrm{~W}$ with area power density of $19 \mathrm{~mW} \cdot \mathrm{cm}^{-2}$ was achieved at an efficiency of $24 \%$, taking a gigantic leap with the output power by orders of magnitude than that of other works. The reported TENG mainly composes of a rotator and a stator as shown in Fig. 9(a). The rotator consists of a total of 60 units with radial array of sectors with equal-degree intervals of $3^{\circ}$. The stator is composed of three terms including an electrification material with fluorinated ethylene propylene (FEP) layer, a layer of electrodes, and a substrate along the vertical direction. The electrode layer is made up of two complementary-patterned electrode networks as the pattern of the rotator's, each network is fabricated by radially-arrayed sectors which are connected with each other at one end to ensure the stationary. Although the high output can be realized, the durable is a critical issue for this structure. To extend the working durability of rotational TENG, long-lifetime TENG without unnecessary electricity loss was achieved through a tactful combination of rectified TENGs in parallel with phase difference and rotational contactseparation mode [55]. The rotational TENG mainly consists of two parts (Fig. 9(A)), a stator with ring structure containing 24 electrodes and a rotor with several push rods. As for the stator, every electrode consists of three layers in total, FEP as dielectric layer, Kapton film as supporting layer, and copper film as conducting layer. Owing to the rotational contactseparation mode, the current output can retain up to $\sim 93 \%$ of its initial performance after 7,200,000 rotations under $2.00 \mathrm{r} \cdot \mathrm{s}^{-1}$ of $1,000 \mathrm{~h}$.

As for a single TENG with approximately microwatt level output power, it can't drive most of the highpower consumption electronics. An effective strategy for improving the output power is fabricating multilayered TENG. The layered TENG can be divided in two forms with symmetrical and alternate layered TENG, both of them have been widely studied for harvesting energy [56-59]. Generally, the presence of parasitic capacitance caused by structure and dimension design is harmful to the output performance of TENG. Aiming to understand how the parasitic capacitance influences the output performance, Yin et al. [60] systematically studied the effects of parasitic capacitance on both of the symmetrical and alternate layered TENG using capacitive models. Both of the different structured TENGs consist of a metal electrode (noted A), dielectric film attached metal electrode (noted B), and Kapton thin film with thickness of $130 \mu \mathrm{m}$ served as substrate film (Fig. 9(c)). For the symmetrical layered TENG (SYM-TENG), the one side of substrate film is an electrode, while the other side is dielectric film attached an electrode, and electrodes on both sides of the Kapton act as output ends without parasitic capacitance effects. As for the 
(A)

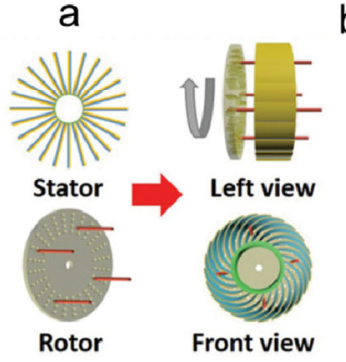

(B) a b

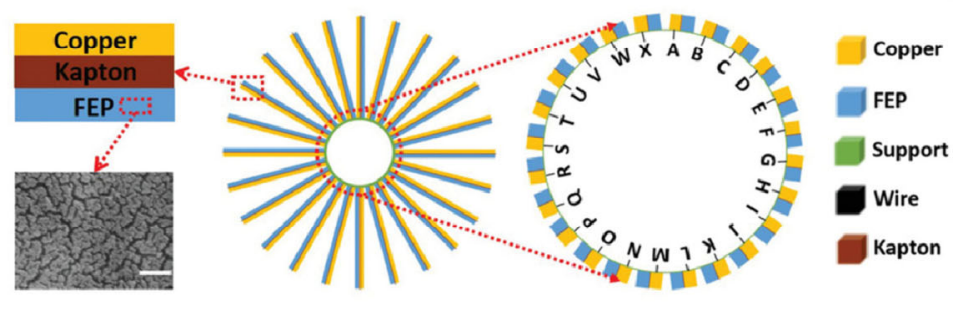

Copper

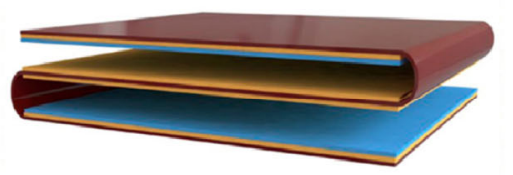

Alternate TENG
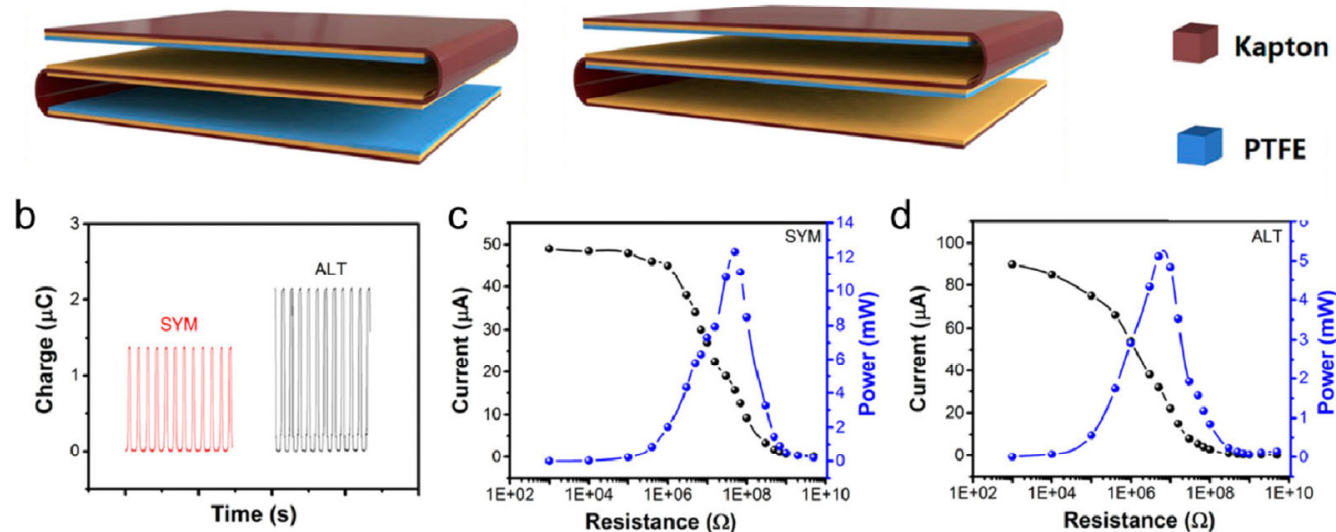

Fig. 9 Improvement of output power by structure design. (A) Design of the rotational TENG with contact-separation mode: (a) schematic description of contact-separation mode TENG and its detailed structure; (b) full size and enlarged detailed version of the stator in contact-separation mode TENG (scale bar: $100 \mathrm{~nm}$ ). Reproduced with permission from Ref. [55]. C WILEY-VCH Verlag GmbH \& Co. $\mathrm{KGaA}$, 2020. (B) Schematic diagram and output performance of both symmetrical and alternate layered TENG: (a-d) schematic diagrams, transferred charge, output current, and power of SYM-TENG and ALT-TENG, respectively. Reproduced with permission from Ref. [60]. (C) American Chemical Society, 2019.

alternate structure (ALT-TENG), both sides of the substrate film are dielectric film connected to a metal electrode, electrodes on both sides are regarded as common outputs, resulting a parasitic capacitor with the Kapton film as dielectric layer. The results are shown that the transferred charge and short-circuit current of ALT-TENG are much higher than those of SYM-TENG, while open circuit voltage of ALT-TENG is less than that of SYM-TENG. It's worth noting that the maximum output power of SYM-TENG is 2.4-fold than that of the ALT-TENG. These results indicate the presence of parasitic capacitance facilitates the improvement of transferred charge, while is harmful to the maximum power density. By understanding the effects of extra parasitic on the output performance of TENGs through experiment and theory, this work provides guidance for designing the TENG and thus enhancing its output performance.

\subsection{Power management}

Considering the AC output with the characteristics of irregular frequency and amplitude, TENG cannot be directly driving most conventional electronics. For transforming the AC output to a regulated and manageable DC output, energy storage units such as batteries and supercapacitors are widely applied by using rectifiers. Owing to the pulsed signal of TENG and the presence of huge impedance mismatch between TENGs and energy storage units, a TENG directly integrated with an energy storage device generally exhibits large power loss and low conversion efficiency. Therefore, advanced power management strategies should be developed to improve the energy conversion efficiency, and the recent progresses are summarized as follows.

One of the most important strategies is impedance 
matching by designing power transformers. For improving the power utilization efficiency by power management, $\mathrm{Pu}$ et al. [61] designed a transformer which can effectively bridge the gap between the battery impedance and matched impedance of the rotating TENG. The equivalent circuit for charging battery by a rotating TENG with the power transformer is shown in Fig. 10(a). In the equivalent circuit model, the TENG is served as a DC voltage source in series with a capacitor $C_{0}\left(Z_{B}=\left(2 \pi f C_{0}\right)^{-1}\right)$, and a battery assumes as an ideal battery in series with an impedance $Z_{B}$. When a transformer with coil ratio of $n$ is applied, the impedance will be reduced to $Z_{1}=n^{-2} Z_{0}$. Therefore, impedance match can be realized by optimization of the transformer. By using the transformer with different coil ratios for battery charging (coil ratio $n=1.0,6.1,24.4$, and 36.7) at $250 \mathrm{rpm}$, the charging current increases with a larger coil ratio, which thus reduces the charging time and enhances the charging power. Through optimizing the coil ratio of a transformer, this study realized a high utilization efficiency of $72.4 \%$ when charging the $\mathrm{LiFePO}_{4}-\mathrm{Li}_{4} \mathrm{Ti}_{5} \mathrm{O}_{12}$ full cell with $n=36.7$. Considering the difference between the output performance and different modes of TENG, this method only can be applied to rotating TENG.

Another excellent work based on impedance matching between TENG and energy storage unit is reported by Niu et al. [59], which was realized by the power management with two stages. Compared to the traditional circuit that achieves the energy conversion and storage for TENG by directly using a bridge rectifier, the improvement of this research is the introduction of additional unit that consists of a small temporary capacitor $\left(C_{\text {temp }}\right)$, two automatic electronic switches $\left(J_{1}, J_{2}\right)$, and a coupled inductor $\left(L_{1}, L_{2}\right)$, as displayed in Fig. 10(b). The power management circuit can be divided into two stages: the first stage

(a)
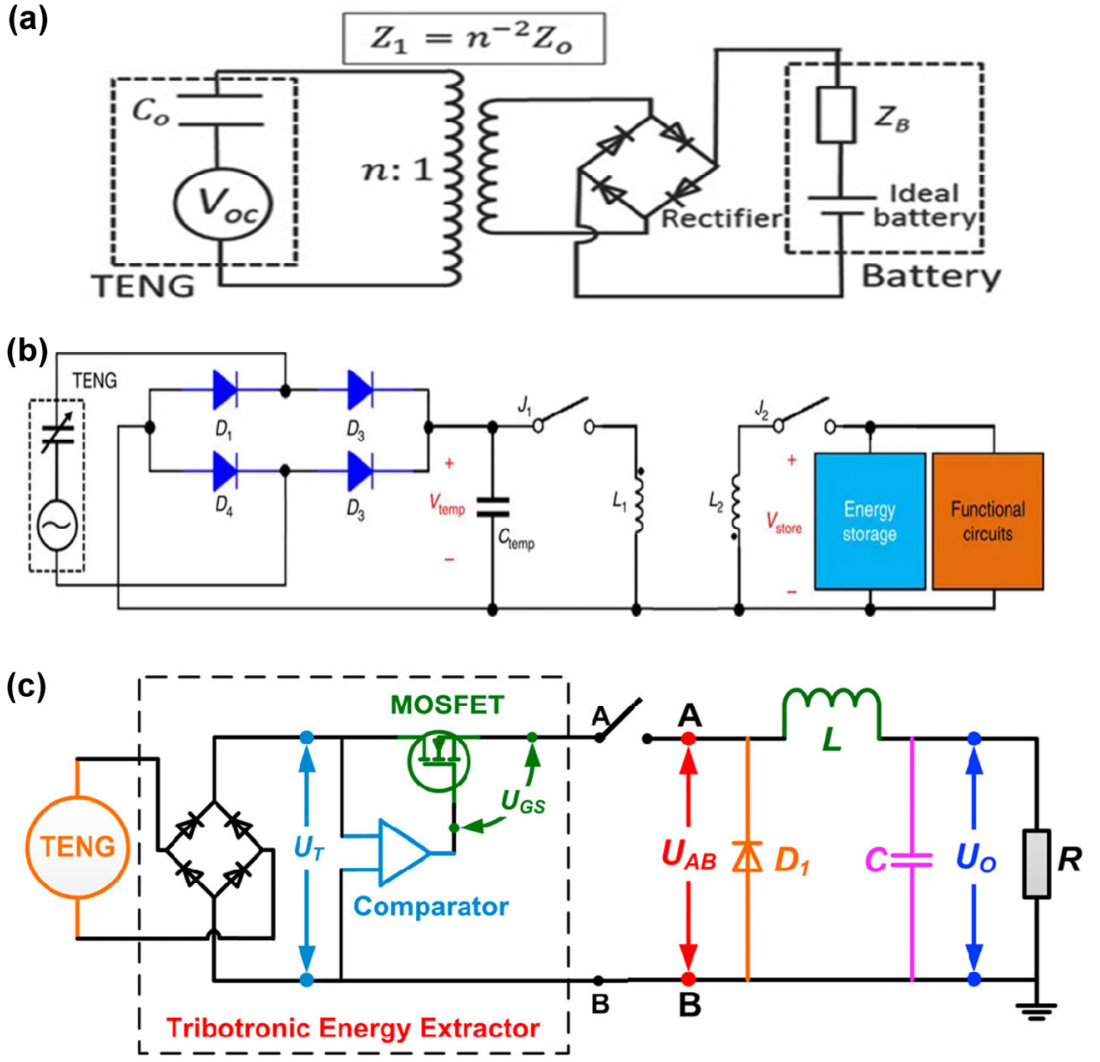

Fig. 10 Power management circuit of TENG. (a) Equivalent circuit for charging battery by a rotating TENG with the power transformer. Reproduced with permission from Ref. [61]. (C) The Authors, 2015. (b) Circuit diagram of the power management circuit based on impedance matching between TENG and energy storage unit. Reproduced with permission from Ref. [59]. (C) Springer Nature, 2015. (c) Schematic circuit diagram of an autonomous power management for TENG by maximizing energy transfer, DC buck conversion, and self-management mechanism. Reproduced with permission from Ref. [62]. C) Elsevier Ltd., 2017. 
is charging the $C_{\text {temp }}$ by the TENG until the optimized charging voltage $\left(V_{\text {opt }}\right)$ is obtained, while the second stage is the energy transformation from the $C_{\text {temp }}$ to the final energy storage unit via $J_{1}, J_{2}$ and $L_{1}, L_{2}$. Using the power management circuit, $90 \%$ board efficiency and $60 \%$ total storage efficiency were realized by optimizing the $C_{\text {temp }}$ and other components, which are improved about two orders of magnitude greater compared with direct charging. Here, the total energy storage efficiency is defined as the ratio of the maximum DC power stored into the storage unit to the maximum AC power delivered to a resistive load. Combining with the power management circuit, a continuous DC electricity of $1.044 \mathrm{~mW}$ was provided by 15-layer TENG with palm tapping, and the reported self-charging unit has demonstrated in powering many traditional electronics, such as a thermometer wearable watch, calculator, and wireless car key.

The above-mentioned work needs power supply for the electronic switches, therefore an advanced work was proposed by $\mathrm{Xi}$ et al. [62] to realize an autonomous power management, which composed of maximized energy transfer, direct current (DC) buck conversion, and self-management mechanism (Fig. 10(c)). The power management circuit can be realized by the following three stages. The first step is achieving the maximum energy transformation for TENG by the aid of a serial switch, and then converts the energy transferred from TENG into a steady and continuous DC output by using a DC buck conversion that consist of parallel freewheeling diode $D_{1}$, a serial inductor $L$, and a parallel capacitor $C$, finally selfmanagement mechanism with an autonomous switch was achieved by a micro-power voltage comparator and a MOSFET. Compared to the previous research for achieving maximized energy output cycle via a parallel switch, this circuit added the switch behind the rectifier in series. By using the power management unit, $80 \%$ energy conversion efficiency for TENG has realized with the matched impedance of the TENG reducing from 35 to $1 \mathrm{M} \Omega$ at a low frequency of $1 \mathrm{~Hz}$.

\section{Recent progresses in TENG applications}

The applications of TENG can be summarized into four major fields: micro/nano power source for small and mobile electronics, self-powered system as an active sensor, integrating many units of TENGs into a network to harvest large-scale blue energy, and HV power source for $\mathrm{HV}$ applications. The recent progresses for TENG will be discussed in this section.

\subsection{Micro/nano power source}

Owing to the merits including light weight, low cost, abundant material, and structural choices, the TENG has demonstrated huge applications as micro/nano power source for self-powered systems by harvesting biomechanical or ambient energy [63-68]. One of recent critical progresses in this field of micro/nano power source is an implantable TENG-based fully implanted symbiotic pacemaker (SPM) proposed by Ouyang et al. [69], which successfully realized energy harvesting and storage by TENG for cardiac pacing on a large-animal model [69]. The SPM composed of three components including the implantable TENG (iTENG) for harvesting energy from cardiac trigger, power management unit (PMU), and pacemaker unit. The first step was to turn off the switch of the PMU, with the electricity generation by iTENG and energy storage in a capacitor of the PMU. Then, a magnet as wireless passive trigger was applied to turn on the switch, the pacemaker unit driven by the electrical energy could generate pacing electrical pulses and control the rate of cardiac contraction (Fig. 11(a)). The iTENG mainly composed of $\mathrm{Al}$ and nanostructured PTFE as two triboelectric layers, supporting the structure with a three-dimensional elastic sponge as a spacer and a memory alloy ribbon as the keel, and two encapsulation layers consisted of a flexible Teflon film and a PDMS film. In the vivo experiment, the output performance of the reported iTENG is more than four times compared with the previous works. As for each cardiac cycle, the harvested energy reached $0.495 \mu \mathrm{J}$, which exceeds the pacing threshold energy of pigs $(0.262 \mu \mathrm{J})$ and humans $(0.377 \mu \mathrm{J})$, demonstrating the progress of TENG as micro/nano power source.

Additionally, as a micro/nano power source, TENG can be used to drive electrochemical reaction for treatment water contaminants. Zhou et al. [70] systematically investigated the electrochemical performance of hexavalent chromium $(\mathrm{Cr}(\mathrm{VI}))$ removal which powered by pulsed output of TENG using the 


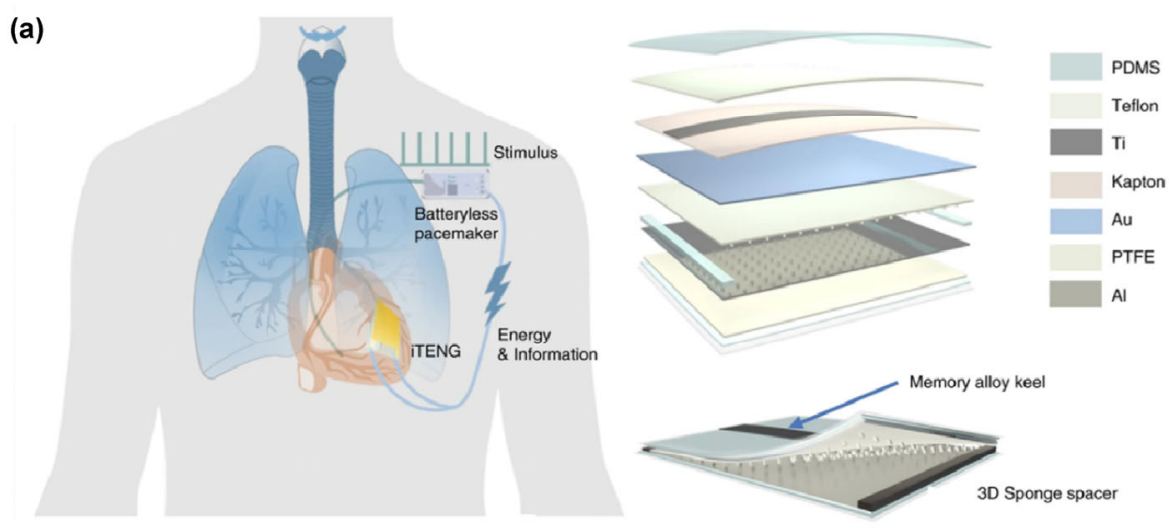

(b)

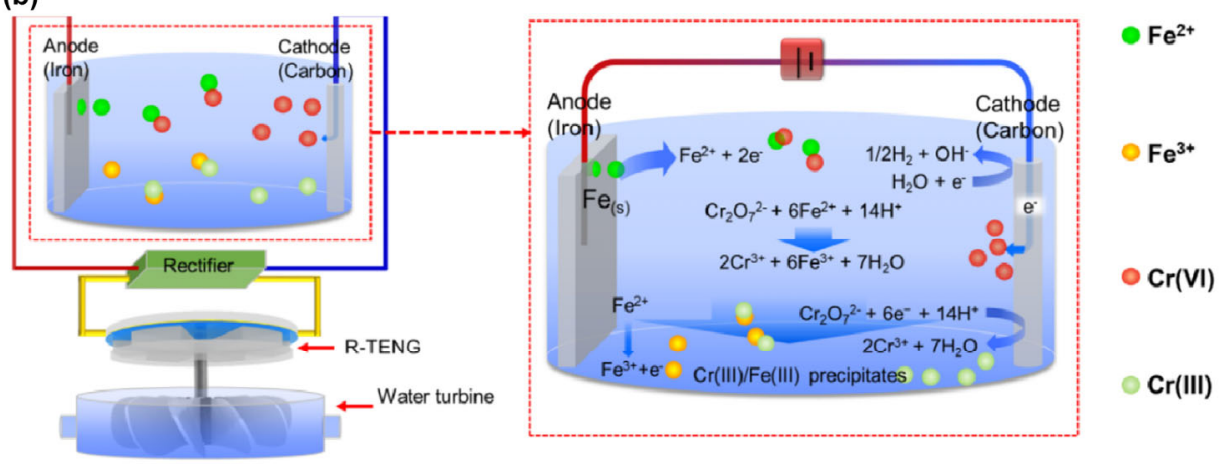

Fig. 11 Application of TENG in micro/nano power source. (A) Illustration of powered cardiac pacemaker via using iTENG. Reproduced with permission from Ref. [69]. C Springer Nature, 2019. (B) Driven electrochemical reaction for removing Cr(VI) by rectified TENG. Reproduced with permission from Ref. [70]. (C) Elsevier Ltd., 2019.

energy harvested from environment (Fig. 11(b)). By optimizing the frequency and on-off ratio of pulse direct current, the removal efficiency of $\mathrm{Cr}(\mathrm{VI})$ can be maximally enhanced by $53.5 \%$ compared to that driven by a continuous direct current under equal amount of electric charges, which is further confirmed by electrochemical experiments driven by layered TENG. The reason is the more production of $\mathrm{Fe}^{2+}$, the better utilization of $\mathrm{Fe}^{2+}$, and the higher ion diffusion rate during the process driven by pulse direct current, where the electrode passivation caused by concentration polarization of the anode region and over potential is reduced. Driven by the rotary-TENG which harvests energy from the flowing water, the electrochemical removal efficiency for contaminant from wastewater was also demonstrated, where heavy metal pollutant such as $\mathrm{Cr}(\mathrm{VI})$ can be sufficiently and continuously removed.

\subsection{Self-powered sensors}

Because the TENG can directly convert mechanical trigger into electric signals, it has been widely studied to be used as self-powered sensor, such as touch sensor [71, 72], acoustic sensors [73, 74], motion and acceleration sensors [75-77], and even chemical sensors [78-80]. With the fast development of robotics, the social interaction and power/energy challenges will be the limitation for its further progress. To address these issues, a self-powered triboelectric auditory system (TAS) based on TENG was fabricated for constructing an electronic hearing system for both robotics and human beings [81]. The triboelectric cochlea device for the TAS is integrated inside the artificial ear. The multilayered TAS mainly composed of a FEP-stick to upper electrode with several hole channels, a certain gap-created spacer with thickness of $\sim 100 \mu \mathrm{m}$, and a Kapton membrane adhered to the bottom electrode. An annular acrylic sheet is served as the outer boundary for the Kapton film, resulting in the free motion for the inner film. During its operation, acoustic wave induces the vibration of Kapton membrane, which leads to the physic contact between the FEP 
and Kapton film, resulting in a potential difference between the two electrodes and finally electric signal output. By adjusting the gap distance, membrane thickness and diameter, TAS achieved an ultrahigh sensitivity with $110 \mathrm{mV} \cdot \mathrm{dB}^{-1}$ and a broad frequency response ranging from 100 to $5,000 \mathrm{~Hz}$, almost across the human hearing range. Furthermore, a tailored frequency response from the sensor itself was realized by optimizing the geometric design of inner boundary architecture, which was demonstrated to be a hearing aid device for recovering hearing-impaired people, thus supplementing human-robot interaction from the auditory sense. This work expresses a prospective application of the TENG-based self-powered sensor for meeting the future challenges of robotics. Inspired by electric eel which can generate high voltages up to $600 \mathrm{~V}$ by stacking thousands of electrolytes in series, a bionic stretchable nanogenerator (BSNG) based on liquid-electrification was proposed for underwater energy harvesting and sensing application (Fig. 12) [82]. Beneficial from numerous electrocytes in series, electric eel can produce electricity with 600 V. Similar to the structure of electrocyte in electric eel, the BSNG consists of two layers including an electrification layer and an induction layer. Specifically, the electrification composed of multiple mechanosensitive channels
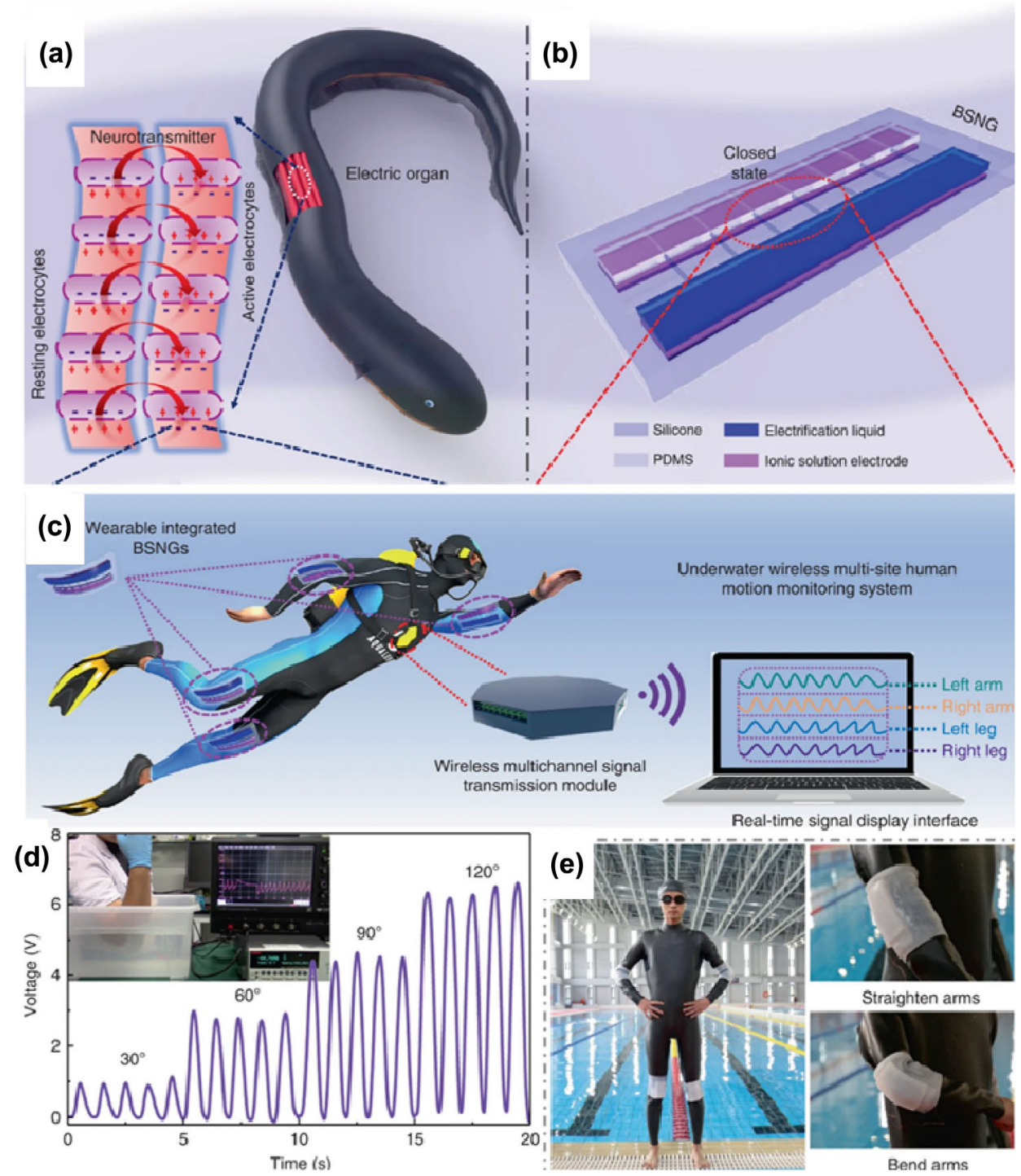

Fig. 12 Application of TENG in self-powered sensors. (a, b) Bionic principle and structure of BSNG, (c) underwater wireless multi-site human motion monitoring system, (d) output performance of BSNG fixed on the elbow, (e) photographs of integrated wearable BSNG worn on the arthrosis of human. Reproduced with permission from Ref. [82]. C Springer Nature, 2019. 
produced by PDMS-silicone double layer structure and a fluid chamber filled with deionized water as electrification liquid, while the induction layer includes two ionic solution electrodes under the channels and chamber of the first layer. Through controlling the gated channel in BSNG by mechanical stress, the circular flowing of electrification liquid was realized, bringing about a continuous alternating electric current. Integration with stretchable silicone, segmented structured of PDMS, liquid electrode, and electrifycation liquid, the BSNG exhibited excellent flexibility, stretchability, and tensile fatigue resistance. Additionally, the silicone layer is regarded as electrostatic shielding layer to ensure the charge transfer process in liquid environment as well as the thick adipose layer of the electric eel, resulting in a good performance of BSNG. With the outstanding performance in liquid environments, BSNG displayed a potential application in powering wearable device applied in moist environment even underwater, which were demonstrated to monitor human body motion underwater and undersea rescue.

\subsection{Blue energy}

Among the application of TENG in harvesting natural mechanical energy from wind [83-86], raindrop [87, $88]$, and ultrasonic $[89,90]$, the wave energy in ocean as blue energy is especially important, owing to the higher efficiency of TENG for harvesting low-frequency vibration energy compared with an electromagnetic generator [91-94]. Compared to other prototypes of TENGs reported for the blue energy, the fully enclosed rolling spherical structure has been identified as the most promising method $[95,96]$. To improve the intimate contact between the core and shell in spherical TENG, a soft-contact model spherical TENG (SS-TENG) is proposed by replacing the hard-dielectric material (PTFE) to a soft core fabricated with liquid/silicone, resulting in 10-fold enhancement to the maximum output charge [97]. The SS-TENG mainly composed of three parts, including a hollow acrylic sphere outside, two copper films as electrodes, and a rolling soft core inside as triboelectric material (Figs. 13(a) and 13(b)). The soft core fabricated by silicone rubber sphere which filled with water. The driving by water waves, the soft rolling sphere contact with the two electrodes with a back and forth motion, resulting in potential difference between the electrodes and finally alternating current in the external load. By optimizing the softness of the silicone defined as the diameter of contact area (Dca), the rolling soft liquid/silicone core with Dca of $3.9 \mathrm{~cm}$ (SS-TENG-2) exhibited an optimum output performance with lower force compared with Dca of $4.8 \mathrm{~cm}$ and large contact area compared with Dca of 3.0 and $0.4 \mathrm{~cm}$ (Fig. 13(c)). Importantly, a maximum transferred charge of $\sim 500 \mathrm{nC}$ was achieved for SS-TENG, which exceeds up 10 times compared to the conventional spherical TENG that PTFE is served as dielectric film. By harvesting the water wave energy, SS-TENG worked in water as a power source can light 26 green LEDs or 56 blue LEDs (Fig. 13(d)). Moreover, the parameters of SS-TENG such as the size of silicone rubber sphere and the volume of water in silicon rubber sphere can be designed to match the outside impact frequency of water wave in ocean. Integrating many of TENG units into a network has been proven to be a promising strategy for harvesting large-scale blue energy. However, the practical application of large-scale TENG units still suffers from the challenge including complicated construction, difficulty of maintenance, failure of connection, and the threats from harsh ocean environment such as storm. As for better adapting the ocean environment, an advanced effort of a self- assembly TENG network based on encapsulated high-performance units was developed for harvesting water wave energy [98]. Because of the self-assembly of the TENG, the network also achieved the abilities including self-healing and reconfiguration. The self-assembly TENG network is mainly composed of an encapsulated spherical TENG with three-dimensional (3D) electrodes and a selfadaptive magnetic joint (SAM-joint). As for the encapsulated spherical TENG unit, a group of plates connects together with each other using copper film to form a pair of $3 \mathrm{D}$ electrode which is sealed by a spherical ball, while FEP pellets are served as triboelectric material which put into the internal channels of the 3D electrode ball (Figs. 13(e) and 13(f)). In addition, SAM-joints with characteristics of a rotatable spherical magnet and a limit block are installed around the shell to facilitate the self-assembly of TENG network. Agitating by water waves, the FEP pellets roll across 

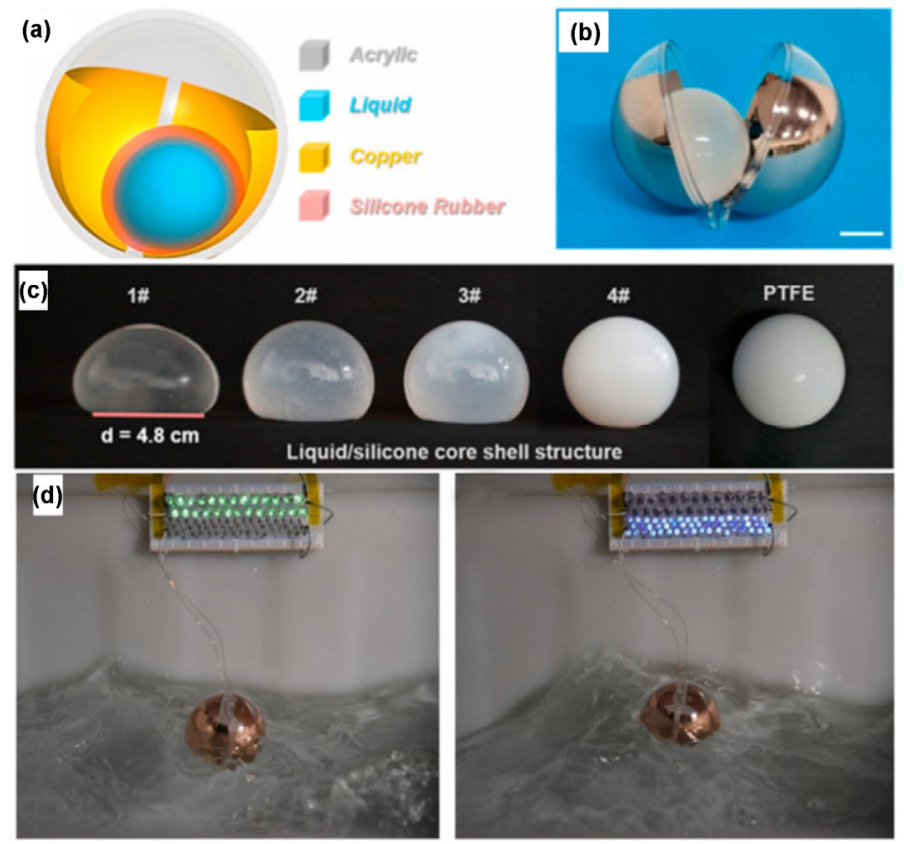

(e)

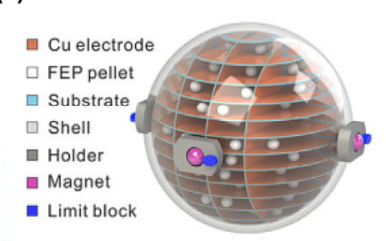

(f)

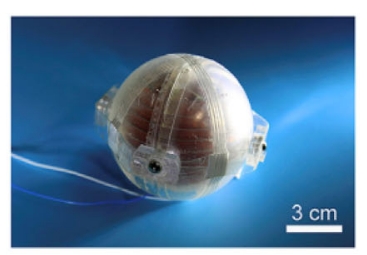

(g)

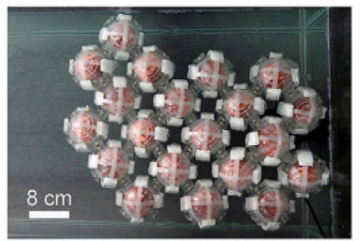

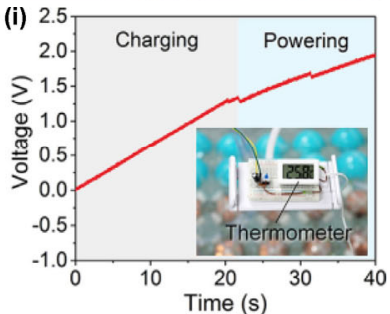

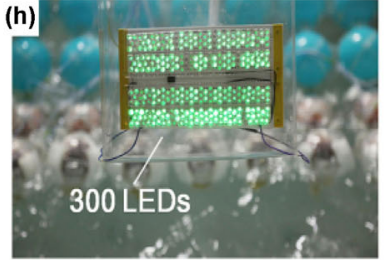

(h)

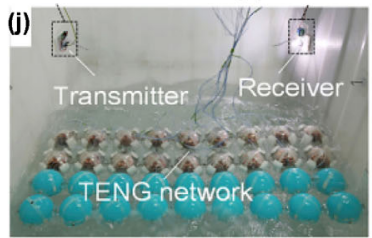

Fig. 13 Application of TENG in blue energy. (a, b) Structural scheme of the soft-contact model spherical TENG, (c) photographs of the flexible rolling sphere with different shell parameters, and (d) output performance of soft-contact model spherical TENG. Reproduced with permission from Ref. [97]. (C) Elsevier Ltd., 2018. (e-g) Schematic structure and photograph of the 3D electrode structured TENG and the self-assembled network, (h) photograph of the self-assembly network to 300 LEDs, thermometer, and the wireless transmitter. Reproduced with permission from Ref. [98]. (c) Elsevier Ltd., 2019.

the internal channels between the two 3D electrodes, generating different charges on the surface of FEP pellets and copper electrode, resulting in electrons flow moving accordingly between the electrodes through an external circuit. By optimizing displacement extent, frequency, and the mass of the FEP pellets in a single TENG unit, an average power density of $8.69 \mathrm{~W} \cdot \mathrm{m}^{-3}$ for a single TENG unit was obtained in air, demonstrating the improvement of output performance by using the 3D electrode structured TENG. Considering the failure of self-assembly TENG which is caused by various magnets meeting with the same pole, the study designed the self-adaptive magnetic joint (SAM-joint) based on the spherical magnet that can rotate freely in the holder. Based on the mechanism of self-adapt of the pole and anisotropic restriction on the degree of freedom, the units of TENG can self-assemble into networks with a certain pattern by introducing different number and distribution of the SAM-joints for each unit (Fig. 13(g)), such as linear chains, a network with a small module of "hollow" hexagon, and a network consisted of a square cluster. The network containing 18 TENG units is demonstrated to harvest water wave energy, which can easily light up 300 LEDs (Fig. 13(h)). The obtained average power of $2.05 \mathrm{~mW} \cdot \mathrm{m}^{-3}$ is more than 18 times compared with the reported ball-shell structured device. Beneficial to the high-power density, the self-assembly TENG network can also be used for self-powered sensing and wireless signal transmission such as self-powered thermometer and turning on a LED bulb through wireless signal transmission (Figs. 13(i) and 13(j)). The self-assembly TENG network provides a promising strategy for large-scale utilization of the blue energy.

\subsection{High voltage power sources}

Since the output performance of TENG exhibits high voltage and low current, it has the potential application in high voltage power sources. Compared to the conventional HV power sources, TENG can easily generate a high voltage up to thousand volts without sophisticated power converters, endowing portability, and lower cost for TENG-based high voltage power source. As a novel HV power source, the high output voltage of TENG was creatively utilized to produce nanoelectrospray ionization for mass spectrometric 
analysis [99], where the limited transferred charges of TENG provided unprecedented control over ion generation. The generated charges from the sliding freestanding TENG were supplied to a nano electrospray ionization emitter for mass spectrometric analysis, and the discrete amount of produced charges guaranteed highly repeatable ionization pulses with minimum sample consumption. The produced TENGtriggered electrospray droplet demonstrated that the onset voltage of the nanoelectrospray ionization emitter was realized. Unprecedented control over the ionization process was enabled by quantized ion pulses of adjustable duration, polarity, and frequency. The high voltage $(5-9 \mathrm{kV})$ of the TENG provided nanoelectrospray ionization with enhanced sensitivity at low concentrations. Finally, a highly sensitive ( $\sim 0.6$ zeptomole) mass spectrometry analysis was demonstrated in analyzing a $10 \mathrm{pg} \cdot \mathrm{ml}^{-1}$ cocaine sample with minimal ion (18 pl per pulse) driven by the sliding freestanding TENG.

The TENG only supplies a low current which is limited by the transferred charges in one cycle, greatly ensuring the safety of personnel and instruments. With the advantages of portability, controllability, safety, and high efficiency, TENGs based HV power sources have been utilized in environmental protection and electrostatic actuation [100-103]. Recently, Cheng
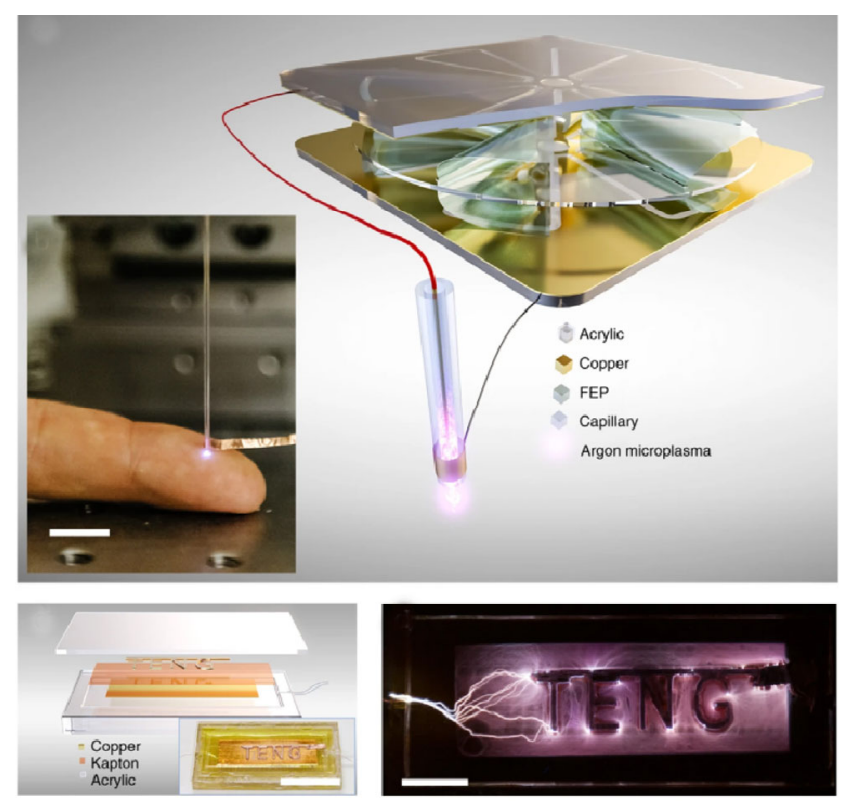

Fig. 14 Application of TENG as high voltage power sources for producing triboelectric microplasma. Reproduced with permission from Ref. [104]. C) Springer Nature, 2018. et al. [104] proposed the concept of triboelectric plasma by integrating TENG with a plasma source, where atmospheric-pressure plasma was directly driven by mechanical stimuli (Fig. 14). The applications of triboelectric plasma in patterned luminescence and plasma surface treatment were demonstrated successfully as a first-step evaluation as well as to prove the system feasibility. This approach might open up possibilities for direct application of TENGs in high-voltage fields, particularly in self-powered plasma.

\section{Summary and perspectives}

Traditionally, we usually refer that the scientific term for TE is called CE, which is about a physical contact between two different materials that would be electrically charged after being separated. However, TE and CE have significant differences. CE occurs just by physical contact of two materials without rubbing one against the other, but TE is usually inseparable involving friction by rubbing two materials one on the other, which involves tribology. TE is a "convolution" of two processes between tribology and CE, so that they are inseparable in conventional understanding. We have recently pointed out that CE is a physics effect in science, while TE is an engineering practice that may involve friction and debris. As for the case of solid-solid, CE is defined as a quantum mechanical electron transfer process that occurs for any material, in any state (solid, liquid, gas), in any application environment, and in a wide range of temperatures up to $\sim 400{ }^{\circ} \mathrm{C}$. Such an effect is universal and fundamentally unique in nature.

Although TE has been known for thousands of years, it has not been used for many positive purposes. Even its basic scientific understanding is lacking. The interest in TE is revised due to the invention of TENG. By using the conjunction of $\mathrm{CE}$ and electrostatic induction, TENG can transfer mechanical energy into electric power, which has been demonstrated to a good application for CE in modern industry. Compared to other energy harvesting technologies, TENG exhibits enormous advantages in extracting kinds of mechanical energy dispersed in our daily life, because of the merits of simple structure, light weight, broad material 
availability, low cost, and high efficiency even at low operation frequency, and thus has been proven to be a promising alternatively method for satisfying the demands of distributed energy for IoTs and network. With the huge progresses of basic principle and output performance for TENG, its major application has extended to four fields including powering small electronic devices for micro/nano power source, building self-powered systems functioning as active sensors for robotics, soft/flexible electronics and artificial intelligence, integrating many units of TENGs into a network for harvesting large- scale blue energy, and applying as HV power source for HV applications. Based on these four major fields, a road map for TENG was proposed in 2018 to identify prioritized directions and provide a timeframe for TENG development (Fig. 15) [105].

Besides the four major research fields, some new horizons for exploring the study and application of TENG are also proposed. Nie et al. [106] reported a liquid-liquid TENG for harvesting mechanical energy forming liquid-liquid interface based on physic contact between two pure liquids. By passing a liquid droplet or other solid objects through a freely suspended liquid membrane, the displacement current on multiphase interface was firstly realized. Liu et al. [107] presented a novel constant current TENG (DC-TENG) by coupling the effects of triboelectrification and electrostatic breakdown. Compared with the conventional TENG limited by electrostatic breakdown, a higher triboelectric charge density of $430 \mu \mathrm{C} \cdot \mathrm{m}^{-2}$ was achieved for the DC-TENG, which was demonstrated to power electronic directly. These two excellent studies open up some new directions for TENG and self-powered systems, which is beneficial for the corresponding fundamental physics study and practical application.

Although the tremendous achievements have been realized in both fundamental understanding and technological improvements, there are some certain issues and problems need to be addressed for the long-term development of TENGs:

1) Further investigating the fundamental physics of CE at solid-solid interfaces, solid-liquid interfaces, and liquid-liquid interfaces, respectively. The current main conclusion is that electron transfer is the dominant mechanism for $\mathrm{CE}$ in solid-solid cases. Recently, the study of CE has been extended to liquidsolid cases. It is revealed that there are both electron transfer and ion transfer in the liquid-solid CE, where electron transfer plays a dominant role in liquid-solid

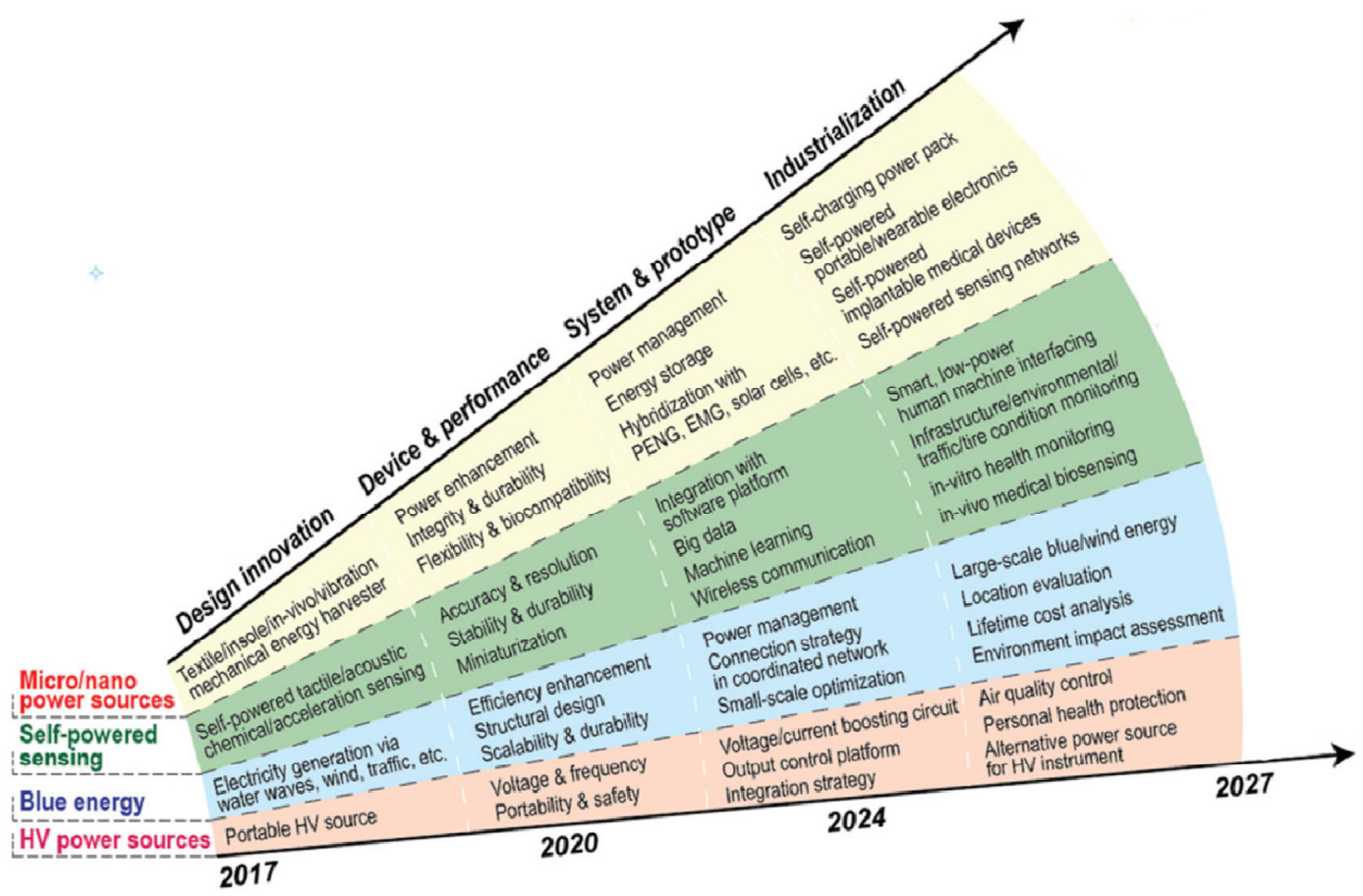

Fig. 15 Road map for the development of TENG. Reproduced with permission from Ref. [105]. C WILEY-VCH Verlag GmbH \& Co. KGaA, 2018. 
CE [108]. Specifically, the CE between hydrophilic surfaces and aqueous solutions is likely dominated by ion transfer, while the CE between hydrophobic surfaces and aqueous solutions is more likely to be dominated by electron transfer [109].

2) Improving the power density of TENG, especially those of TENGs in the wearable devices. Various methods such as selecting new materials, enhancing the contact intimacy, optimizing the working environment, and utilizing a thin film as dielectric layer would be promising choices. As for solid-solid contact devices of TENG, interface adhesion, material transfer, and wear may occur during the long-term and high frequency operation, therefore fabricating materials with robust mechanical durability and stability might be an alternative method to solve the problem. As for the liquid-solid device, the dissolution of electrodes may cause some lifetime problems, thus good encapsulation technology and superhydrophobic surface may prolong the lifetime of TENG.

3) Enhancing the durability of TENG with high power generation. Long-term cycling stability power generation is always a vital issue for the large-scale power application of TENG. Generally, contactseparation mode has long working life. New structure design, such as rotational contact-separation mode, is desirable to achieve long-lifetime TENG to harvest rotational mechanical energy.

4) Promoting the development of TENG for harvesting the blue energy. Although large efforts have been devoted to enhancing the energy harvested efficiency, adaptive capacity in actual marine environment should be addressed in the further study. To satisfy the high performance and adaptive capacity at the same time, an appropriate platform integrated with TENG would be a promising method.

\section{Acknowledgements}

This research was supported by the National Key $\mathrm{R}$ \& D Project from Minister of Science and Technology (2016YFA0202704), National Natural Science Foundation of China (Grant Nos. 61774016, 21773009, 51432005, 5151101243, and 51561145021), and China Postdoctoral Science Foundation (2019M660587). The authors thank our group members and collaborators for their contribution to the development of TENG.

Open Access This article is licensed under a Creative Commons Attribution 4.0 International License, which permits use, sharing, adaptation, distribution and reproduction in any medium or format, as long as you give appropriate credit to the original author(s) and the source, provide a link to the Creative Commons licence, and indicate if changes were made.

The images or other third party material in this article are included in the article's Creative Commons licence, unless indicated otherwise in a credit line to the material. If material is not included in the article's Creative Commons licence and your intended use is not permitted by statutory regulation or exceeds the permitted use, you will need to obtain permission directly from the copyright holder.

To view a copy of this licence, visit http://creativecommons.org/licenses/by/4.0/.

\section{References}

[1] Wang Z L, Wang A C. On the origin of contact-electrification. Mater Today 30: 34-51 (2019)

[2] Shaw P E. The electrical charges from like solids. Nature 118(2975): 659-660 (1926)

[3] Fan F R, Tian Z Q, Wang Z L. Flexible triboelectric generator. Nano Energy 1(2): 328-334 (2012)

[4] Wang Z L. Triboelectric nanogenerators as new energy technology and self-powered sensors-principles, problems and perspectives. Faraday Discuss 176: 447-458 (2014)

[5] Lin L, Wang S H, Xie Y N, Jing Q S, Niu S M, Hu Y F, Wang Z L. Segmentally structured disk triboelectric nanogenerator for harvesting rotational mechanical energy. Nano Lett 13(6): 2916-2923 (2013)

[6] Luo J J, Wang Z M, Xu L, Wang A C, Han K, Jiang T, Lai Q S, Bai Y, Tang W, Fan F R, et al. Flexible and durable wood-based triboelectric nanogenerators for self-powered sensing in athletic big data analytics. Nat Commun 10(1): 5147 (2019)

[7] Wang S H, Lin L, Xie Y N, Jing Q S, Niu S M, Wang Z L. Sliding-triboelectric nanogenerators based on in-plane chargeseparation mechanism. Nano Lett 13(5): 2226-2233 (2013)

[8] Zhu G, Peng B, Chen J, Jing Q S, Wang Z L. Triboelectric nanogenerators as a new energy technology: From fundamentals, devices, to applications. Nano Energy 14: 126-138 (2015) 
[9] Zi Y L, Wang Z L. Nanogenerators: An emerging technology towards nanoenergy. APL Mater 5(7): 074103 (2017)

[10] Yi F, Wang X F, Niu S M, Li S M, Yin Y J, Dai K R, Zhang G J, Lin L, Wen Z, Guo H Y, et al. A highly shape-adaptive, stretchable design based on conductive liquid for energy harvesting and self-powered biomechanical monitoring. Sci Adv 2(6): e1501624 (2016)

[11] Chen J, Huang Y, Zhang N N, Zou H Y, Liu R Y, Tao C Y, Fan $X$, Wang $Z$ L. Micro-cable structured textile for simultaneously harvesting solar and mechanical energy. Nat Energy 1(10): 16138 (2016)

[12] Wang Z L, Chen J, Lin L. Progress in triboelectric nanogenerators as a new energy technology and self-powered sensors. Energy Environ Sci 8(8): 2250-2282 (2015)

[13] Wang Z L. Entropy theory of distributed energy for internet of things. Nano Energy 58: 669-672 (2019)

[14] Pu X J, Guo H Y, Chen J, Wang X, Xi Y, Hu C G, Wang $\mathrm{Z}$ L. Eye motion triggered self-powered mechnosensational communication system using triboelectric nanogenerator. Sci Adv 3(7): e1700694 (2017)

[15] Hinchet R, Yoon H J, Ryu H, Kim M K, Choi E K, Kim D S, Kim S W. Transcutaneous ultrasound energy harvesting using capacitive triboelectric technology. Science 365(6452): 491-494 (2019)

[16] Pu X, Liu M M, Chen X Y, Sun J M, Du C H, Zhang Y, Zhai J Y, Hu W G, Wang Z L. Ultrastretchable, transparent triboelectric nanogenerator as electronic skin for biomechanical energy harvesting and tactile sensing. Sci $A d v$ 3(5): e1700015 (2017)

[17] Zheng Q, Zou Y, Zhang Y L, Liu Z, Shi B J, Wang X X, Jin Y M, Ouyang H, Li Z, Wang Z L. Biodegradable triboelectric nanogenerator as a life-time designed implantable power source. Sci Adv 2(3): e1501478 (2016)

[18] Chen J, Wang Z L. Reviving vibration energy harvesting and self-powered sensing by a triboelectric nanogenerator. Joule 1(3): 480-521 (2017)

[19] Fan F R, Tang W, Wang Z L. Flexible nanogenerators for energy harvesting and self-powered electronics. Adv Mater 28(22): 4283-4305 (2016)

[20] Xiao T X, Liang X, Jiang T, Xu L, Shao J J, Nie J H, Bai Y, Zhong W, Wang Z L. Spherical triboelectric nanogenerators based on spring-assisted multilayered structure for efficient water wave energy harvesting. Adv Funct Mater 28: 1802634 (2018)

[21] Wang Z L. New wave power. Nature 542: 159-160 (2017)

[22] Liang X, Jiang T, Liu G X, Feng Y W, Zhang C, Wang Z L. Spherical triboelectric nanogenerator integrated with power management module for harvesting multidirectional water wave energy. Energy Environ Sci 13(1): 277-285 (2020)

[23] Jiang T, Yao Y Y, Xu L, Zhang L M, Xiao T X, Wang Z L. Spring-assisted triboelectric nanogenerator for efficiently harvesting water wave energy. Nano Energy 31: 560-567 (2017)

[24] Wu C S, Liu R Y, Wang J, Zi Y L, Lin L, Wang Z L. A spring-based resonance coupling for hugely enhancing the performance of triboelectric nanogenerators for harvesting low-frequency vibration energy. Nano Energy 32: 287-293 (2017)

[25] Lin S Q, Xu C, Xu L, Wang Z L. The overlapped electroncloud model for electron transfer in contact electrification. Adv Funct Mater 30(11): 1909724 (2020)

[26] Baytekin H T, Patashinski A Z, Branicki M, Baytekin B, Soh S, Grzybowski B A. The Mosaic of surface charge in contact electrification. Science 333(6040): 308-312 (2011)

[27] Xu C, Zi Y L, Wang A C, Zou H Y, Dai Y J, He X, Wang P H, Wang Y C, Feng P Z, Li D W, et al. On the electrontransfer mechanism in the contact-electrification effect. $A d v$ Mater 30(15): 1706790 (2018)

[28] Lowell J, Akande A R. Contact electrification-why is it variable? J Phys D: Appl Phys 21(1): 125-137 (1988)

[29] Wang Z L. On Maxwell's displacement current for energy and sensors: The origin of nanogenerators. Mat Today 20(2): 74-82 (2017)

[30] Wang Z L. On the first principle theory of nanogenerators from Maxwell's equations. Nano Erengy 68: 104272 (2019)

[31] Zhu G, Pan C F, Guo W X, Chen C Y, Zhou Y S, Yu R M, Wang Z L. Triboelectric-generator-driven pulse electrodeposition for micropatterning. Nano Lett 12(9): 4960-4965 (2012)

[32] Wang S H, Lin L, Wang Z L. Nanoscale triboelectriceffect-enabled energy conversion for sustainably powering portable electronics. Nano Lett 12(12): 6339-6346 (2012)

[33] Wang S H, Lin L, Xie Y N, Jing Q S, Niu S M, Wang Z L. Sliding-triboelectric nanogenerators based on in-plane charge-separation mechanism. Nano Lett 13(5): 2226-2233 (2013)

[34] Zhu G, Chen J, Liu Y, Bai P, Zhou Y S, Jing Q S, Pan C F, Wang Z L. Linear-grating triboelectric generator based on sliding electrification. Nano Lett 13(5): 2282-2289 (2013)

[35] Niu S M, Liu Y, Wang S H, Lin L, Zhou Y S, Hu Y F, Wang $Z$ L. Theoretical investigation and structural optimization of single-electrode triboelectric nanogenerators. Adv Funct Mater 24(22): 3332-3340 (2014)

[36] Yang Y, Zhou Y S, Zhang H L, Liu Y, Lee S, Wang Z L. A single-electrode based triboelectric nanogenerator as selfpowered tracking system. Adv Mater 25(45): 6594-6601 (2013) 
[37] Wang S H, Xie Y N, Niu S M, Lin L, Wang Z L. Freestanding triboelectric-layer-based nanogenerators for harvesting energy from a moving object or human motion in contact and non-contact modes. Adv Mater 26(18): 2818-2824 (2014)

[38] Zi Y L, Niu S M, Wang J, Wen Z, Tang W, Wang Z L. Standards and figure-of-merits for quantifying the performance of triboelectric nanogenerators. Nat Commun 6: 8376 (2015)

[39] Zhang X S, Han M D, Wang R X, Meng B, Zhu F Y, Sun X M, Hu W, Wang W, Li Z H, Zhang H X. High-performance triboelectric nanogenerator with enhanced energy density based on single-step fluorocarbon plasma treatment. Nano Energy 4: 123-131 (2014)

[40] Lin Z H, Xie Y N, Yang Y, Wang S H, Zhu G, Wang $Z$ L. Enhanced triboelectric nanogenerators and triboelectric nanosensor using chemically modified $\mathrm{TiO}_{2}$ nanomaterials. ACS Nano 7(5): 4554-4560 (2013)

[41] Han S S, Ko Y J, Kim D Y, Jung J H. Enhanced triboelectric charge through a facile hydrothermal treatment of electrode. Curr Appl Phys 16(10): 1364-1368 (2016)

[42] Cui N Y, Gu L, Lei Y M, Liu J M, Qin Y, Ma X H, Hao Y, Wang Z L. Dynamic behavior of the triboelectric charges and structural optimization of the friction layer for a triboelectric nanogenerator. ACS Nano 10(6): 6131-6138 (2016)

[43] Wang S H, Zi Y L, Zhou Y S, Li S M, Fan F R, Lin L, Wang Z L. Molecular surface functionalization to enhance the power output of triboelectric nanogenerators. $J$ Mater Chem A 4(10): 3728-3734 (2016)

[44] Zou H Y, Zhang Y, Guo L T, Wang P H, He X, Dai G Z, Zheng H W, Chen C Y, Wang A C, Xu C, et al. Quantifying the triboelectric series. Nat Commun 10(1): 1427 (2019)

[45] Wang J, Wen Z, Zi Y L, Zhou P F, Lin J, Guo H Y, Xu Y L, Wang Z L. All-plastic-materials based self-charging power system composed of triboelectric nanogenerators and supercapacitors. Adv Funct Mater 26(7): 1070-1076 (2016)

[46] Wang J, Li S M, Yi F, Zi Y L, Lin J, Wang X F, Xu Y L, Wang Z L. Sustainably powering wearable electronics solely by biomechanical energy. Nat Commun 7: 12744 (2016)

[47] Lin S Q, Xu L, Tang W, Chen X Y, Wang Z L. Electron transfer in nano-scale contact electrification: Atmosphere effect on the surface states of dielectrics. Nano Energy $\mathbf{6 5}$ : 103956 (2019)

[48] Wang J, Wu C S, Dai Y J, Zhao Z H, Wang A, Zhang T J, Wang Z L. Achieving ultrahigh triboelectric charge density for efficient energy harvesting. Nat Commun 8(1): 88 (2017)

[49] Zhang C L, Zhou L L, Cheng P, Yin X, Liu D, Li X Y, Guo H Y, Wang Z L, Wang J. Surface charge density of triboelectric nanogenerators: Theoretical boundary and optimization methodology. Appl Mater Today 18: 100496
(2020)

[50] Wang S H, Xie Y N, Niu S M, Lin L, Liu C, Zhou Y S, Wang Z L. Maximum surface charge density for triboelectric nanogenerators achieved by ionized-air injection: Methodology and theoretical understanding. Adv Mater 26(39): 6720-6728 (2014)

[51] Cheng L, Xu Q, Zheng Y B, Jia X F, Qin Y. A selfimproving triboelectric nanogenerator with improved charge density and increased charge accumulation speed. Nat Commun 9: 3773 (2018)

[52] Xu L, Bu T Z, Yang X D, Zhang C, Wang Z L. Ultrahigh charge density realized by charge pumping at ambient conditions for triboelectric nanogenerators. Nano Energy 49: 625-633 (2018)

[53] Liu W L, Wang Z, Wang G, Liu G L, Chen J, Pu X J, Xi Y, Wang $\mathrm{X}$, Guo $\mathrm{H} \mathrm{Y}, \mathrm{Hu} \mathrm{C} \mathrm{G}$, et al. Integrated charge excitation triboelectric nanogenerator. Nat Commun 10: 1426 (2019)

[54] Zhu G, Chen J, Zhang T J, Jing Q S, Wang Z L. Radialarrayed rotary electrification for high performance triboelectric generator. Nat Commun 5: 3426 (2014)

[55] Li X Y, Yin X, Zhao Z H, Zhou L L, Liu D, Zhang C L, Zhang C G, Zhang W, Li S X, Wang J, et al. Long-lifetime triboelectric nanogenerator operated in conjunction modes and low crest factor. Adv Energy Mater 10(7): 903024 (2020)

[56] Peng J, Kang S D, Snyder G J. Optimization principles and the figure of merit for triboelectric generators. Sci $\operatorname{Adv} \mathbf{3}(12)$ : eaap8576 (2017)

[57] Niu S M, Wang Z L. Theoretical systems of triboelectric nanogenerators. Nano Energy 14: 161-192 (2015)

[58] Liu R Y, Wang J, Sun T, Wang M J, Wu C S, Zou H Y, Song T, Zhang X H, Lee S T, Wang Z L, et al. Silicon nanowire/polymer hybrid solar cell-supercapacitor: A self-charging power unit with a total efficiency of $10.5 \%$. Nano Lett 17(7): 4240-4247 (2017)

[59] Niu S M, Wang X F, Yi F, Zhou Y S, Wang Z L. A universal self-charging system driven by random biomechanical energy for sustainable operation of mobile electronics. Nat Commun 6: 8975 (2015)

[60] Yin X, Liu D, Zhou L L, Li X Y, Zhang C L, Cheng P, Guo H Y, Song W X, Wang J, Wang Z L. Structure and dimension effects on the performance of layered triboelectric nanogenerators in contact-separation mode. ACS Nano 13(1): 698-705 (2019)

[61] Pu X, Liu M M, Li L X, Zhang C, Pang Y K, Jiang C Y, Shao L H, Hu W G, Wang Z L. Efficient charging of Li-ion batteries with pulsed output current of triboelectric nanogenerators. Adv Sci 3(4): 1500255 (2016) 
[62] Xi F B, Pang Y K, Li W, Jiang T, Zhang L M, Guo T, Liu $\mathrm{G} \mathrm{X}$, Zhang $\mathrm{C}$, Wang $\mathrm{Z} \mathrm{L}$. Universal power management strategy for triboelectric nanogenerator. Nano Energy 37: 168-176 (2017)

[63] Yang W Q, Chen J, Zhu G, Yang J, Bai P, Su Y J, Jing Q S, Cao X, Wang Z L. Harvesting energy from the natural vibration of human walking. ACS Nano 7(12): 11317-11324 (2013)

[64] Yang J, Chen J, Liu Y, Yang W Q, Su Y J, Wang Z L. Triboelectrification-based organic film nanogenerator for acoustic energy harvesting and self-powered active acoustic sensing. ACS Nano 8(3): 2649-2657 (2014)

[65] Fan X, Chen J, Yang J, Bai P, Li Z L, Wang Z L. Ultrathin, rollable, paper-based triboelectric nanogenerator for acoustic energy harvesting and self-powered sound recording. ACS Nano 9(4): 4236-4243 (2015)

[66] Yi F, Wang X F, Niu S M, Lin S M, Yin Y J, Dai K R, Zhang G J, Lin L, Wen Z, Guo H Y, et al. A highly shapeadaptive, stretchable design based on conductive liquid for energy harvesting and self-powered biomechanical monitoring. Sci Adv 2(6): e1501624 (2016)

[67] Pu X, Liu M M, Chen X Y, Sun J M, Du C H, Zhang Y, Zhai J Y, Hu W G, Wang Z L. Ultrastretchable, transparent triboelectric nanogenerator as electronic skin for biomechanical energy harvesting and tactile sensing. Sci Adv 3(5): e1700015 (2017)

[68] Zheng Q, Zou Y, Zhang Y L, Liu Z, Shi B J, Wang X X, Jin Y M, Ouyang H, Li Z, Wang Z L. Biodegradable triboelectric nanogenerator as a life-time designed implantable power source. Sci Adv 2(3): e1501478 (2016)

[69] Ouyang H, Liu Z, Li N, Shi B J, Zou Y, Xie F, Ma Y, Li Z, Li H, Zheng Q, et al. Symbiotic cardiac pacemaker. Nat Commun 10(1): 1821 (2019)

[70] Zhou L L, Liu D, Li S X, Yin X, Zhang C L, Li X Y, Zhang C G, Zhang W, Cao X, Wang J, et al. Effective removing of hexavalent chromium from wasted water by triboelectric nanogenerator driven self-powered electrochemical systemWhy pulsed DC is better than continuous DC? Nano Energy 64: 103915 (2019)

[71] Yang Y, Zhang H L, Lin Z H, Zhou Y S, Jing Q S, Su Y J, Yang J, Chen J, Hu C G, Wang Z L. Human skin based triboelectric nanogenerators for harvesting biomechanical energy and as self-powered active tactile sensor system. ACS Nano 7(10): 9213-9222 (2013)

[72] Zhu G, Yang W Q, Zhang T J, Jing Q S, Chen J, Zhou Y S, Bai P, Wang Z L. Self-powered, ultrasensitive, flexible tactile sensors based on contact electrification. Nano Lett 14(6): 3208-3213 (2014)

[73] Yang J, Chen J, Liu Y, Yang W Q, Su Y J, Wang Z L.
Triboelectrification-based organic film nanogenerator for acoustic energy harvesting and self-powered active acoustic sensing. ACS Nano 8(3): 2649-2657 (2014)

[74] Yu A F, Song M, Zhang Y, Zhang Y, Chen L B, Zhai J Y, Wang Z L. Self-powered acoustic source locator in underwater environment based on organic film triboelectric nanogenerator. Nano Res 8(3): 765-773 (2015)

[75] Zhou Y S, Zhu G, Niu S M, Liu Y, Bai P, Jing Q S, Wang Z L. Nanometer resolution self-powered static and dynamic motion sensor based on micro-grated triboelectrification. Adv Mater 26(11): 1719-1724 (2014)

[76] Yi F, Lin L, Niu S M, Yang J, Wu W Z, Wang S H, Liao Q L, Zhang Y, Wang Z L. Self-powered trajectory, velocity, and acceleration tracking of a moving object/body using a triboelectric sensor. Adv Funct Mater 24(47): 7488-7494 (2014)

[77] Wu C S, Wang X, Lin L, Guo H Y, Wang Z L. Paper-based triboelectric nanogenerators made of stretchable interlocking kirigami patterns. ACS Nano 10(4): 4652-4659 (2016)

[78] Li Z L, Chen J, Yang J, Su Y J, Fan X, Wu Y, Yu C W, Wang Z L. $\beta$-cyclodextrin enhanced triboelectrification for self-powered phenol detection and electrochemical degradation. Energy Environ Sci 8(3): 887-896 (2015)

[79] Wen Z, Chen J, Yeh M H, Guo H Y, Li Z L, Fan X, Zhang $\mathrm{T} \mathrm{J}$, Zhu L P, Wang $\mathrm{Z}$ L. Blow-driven triboelectric nanogenerator as an active alcohol breath analyzer. Nano Energy 16: 38-46 (2015)

[80] Zhang H L, Yang Y, Su Y J, Chen J, Hu C G, Wu Z K, Liu Y, Wong C P, Bando Y, Wang Z L. Triboelectric nanogenerator as self-powered active sensors for detecting liquid/gaseous water/ethanol. Nano Energy 2(5): 693-701 (2013)

[81] Guo H Y, Pu X J, Chen J, Meng Y, Yeh M H, Liu G L, Tang Q, Chen B D, Liu D, Qi S, et al. A highly sensitive, self-powered triboelectric auditory sensor for social robotics and hearing aids. Sci Robot 3(20): eaat2516 (2018)

[82] Zou Y, Tan P C, Shi B J, Ouyang H, Jiang D J, Liu Z, Li H, Yu M, Wang C, Qu X C, et al. A bionic stretchable nanogenerator for underwater sensing and energy harvesting. Nat Commun 10: 2695 (2019)

[83] Yang Y, Zhu G, Zhang H L, Chen J, Zhong X D, Lin Z H, Su Y J, Bai P, Wen X N, Wang Z L. Triboelectric nanogenerator for harvesting wind energy and as self-powered wind vector sensor system. ACS Nano 7(10): 9461-9468 (2013)

[84] Bae J, Lee J, Kim S M, Ha J, Lee B S, Park Y J, Choong C, Kim J B, Wang Z L, Kin H Y, et al. Flutter-driven triboelectrification for harvesting wind energy. Nat Commun 5(1): 4929 (2014) 
[85] Wang J, Ding W B, Pan L, Wu C S, Yu H, Yang L J, Liao R J, Wang Z L. Self-powered wind sensor system for detecting wind speed and direction based on a triboelectric nanogenerator. ACS Nano 12(4): 3954-3963 (2018)

[86] Chen B, Yang Y, Wang Z L. Scavenging wind energy by triboelectric nanogenerators. Adv Energy Mater 8(10): 1702649 (2018)

[87] Zheng L, Lin Z H, Cheng G, Wu W Z, Wen X N, Lee S, Wang Z L. Silicon-based hybrid cell for harvesting solar energy and raindrop electrostatic energy. Nano Energy 9: 291-300 (2014)

[88] Zhu H R, Tang W, Gao C Z, Han Y, Li T, Cao X, Wang Z L. Self-powered metal surface anti-corrosion protection using energy harvested from rain drops and wind. Nano Energy 14: 193-200 (2015)

[89] Xi Y, Wang J, Zi Y L, Li X G, Han C B, Cao X, Hu C G, Wang $Z$ L. High efficient harvesting of underwater ultrasonic wave energy by triboelectric nanogenerator. Nano Energy 38: 101-108 (2017)

[90] Zhao H F, Xiao X, Xu P, Zhao T C, Song L G, Pan X X, Mi J C, Xu M Y, Wang Z L. Dual-tube helmholtz resonator-based triboelectric nanogenerator for highly efficient harvesting of acoustic energy. Adv Energy Mater 9(46): 1902824 (2019)

[91] Zi Y L, Guo H Y, Wen Z, Yeh M H, Hu C G, Wang Z L. Harvesting low-frequency $(<5 \mathrm{~Hz})$ irregular mechanical energy: A possible killer application of triboelectric nanogenerator. ACS Nano 10(4): 4797-4805 (2016)

[92] Wen X N, Yang W Q, Jing Q S, Wang Z L. Harvesting broadband kinetic impact energy from mechanical triggering/ vibration and water waves. ACS Nano 8(7): 7405-7412 (2014)

[93] Zhang L M, Han C B, Jiang T, Zhou T, Li X H, Zhang C, Wang Z L. Multilayer wavy-structured robust triboelectric nanogenerator for harvesting water wave energy. Nano Energy 22: 87-94 (2016)

[94] Leung S F, Fu H C, Zhang M L, Hassan A H, Jiang T, Salama K N, Wang Z L, He J H. Blue energy fuels: Converting ocean wave energy to carbon-based liquid fuels via $\mathrm{CO}_{2}$ reduction. Energy Environ Sci, in press, DOI 10.1039/c9ee03566d.

[95] Yang Y, Zhang H L, Liu R Y, Wen X N, Hou T C, Wang Z L. Fully enclosed triboelectric nanogenerators for applications in water and harsh environments. Adv Energy Mater 3(12): 1563-1568 (2013)

[96] Wang X F, Niu S M, Yin Y J, Yi F, You Z, Wang Z L. Triboelectric nanogenerator based on fully enclosed rolling spherical structure for harvesting low-frequency water wave energy. Adv Energy Mater 5(24): 1501467 (2015)

[97] Cheng P, Guo H Y, Wen Z, Zhang C L, Yin X, Li X Y, Liu D, Song W X, Sun X H, Wang J, et al. Largely enhanced triboelectric nanogenerator for efficient harvesting of water wave energy by soft contacted structure. Nano Energy 57: 432-439 (2019)

[98] Yang X D, Xu L, Lin P, Zhong W, Bai Y, Luo J J, Chen J, Wang Z L. Macroscopic self-assembly network of encapsulated high-performance triboelectric nanogenerators for water wave energy harvesting. Nano Energy 60: 404-412 (2019)

[99] Li A Y, Zi Y L, Guo H Y, Wang Z L, Fernández F M. Triboelectric nanogenerators for sensitive nano-coulomb molecular mass spectrometry. Nat Nanotechnol 12(5): 481-487 (2017)

[100] Chen X Y, Wu Y L, Yu A F, Xu L, Zheng L, Liu Y S, Li H X, Wang Z L. Self-powered modulation of elastomeric optical grating by using triboelectric nanogenerator. Nano Energy 38: 91-100 (2017)

[101] Li C J, Yin Y Y, Wang B, Zhou T, Wang J N, Luo J J, Tang W, Cao R, Yuan Z Q, Li N W, et al. Self-powered electrospinning system driven by a triboelectric nanogenerator. ACS Nano 11(10): 10439-10445 (2017)

[102] Zi Y L, Wu C S, Ding W B, Wang X F, Dai Y J, Cheng J, Wang J Y, Wang Z J, Wang Z L. Field Emission of electrons powered by a triboelectric nanogenerator. $A d v$ Funct Mater 28(21): 1800610 (2018)

[103] Yang H, Pang Y K, Bu T Z, Liu W B, Luo J J, Jiang D D, Zhang C, Wang Z L. Triboelectric micromotors actuated by ultralow frequency mechanical stimuli. Nat Commun 10: 2309 (2019)

[104] Cheng J, Ding W B, Zi Y L, Lu Y J, Ji L H, Liu F, Wu C S, Wang Z L. Triboelectric microplasma powered by mechanical stimuli. Nat Commum 9(1): 3733 (2018)

[105] Wu C S, Wang A C, Ding W B, Guo H Y, Wang Z L. Triboelectric nanogenerator: A foundation of the energy for the new era. Adv Energy Mater 9(1): 1802906 (2019)

[106] Nie J H, Wang Z M, Ren Z W, Li S Y, Chen X Y, Wang Z L. Power generation from the interaction of a liquid droplet and a liquid membrane. Nat Commun 10: 2264 (2019)

[107] Liu D, Yin X, Guo H Y, Zhou L L, Li X Y, Zhang C L, Wang J, Wang Z L. A constant current triboelectric nanogenerator arising from electrostatic breakdown. Sci Adv 5(4): eaav6437 (2019)

[108] Nie J H, Ren Z W, Xu L, Lin S Q, Zhan F, Chen X Y, Wang $Z$ L. Probing contact-electrification-induced electron and ion transfers at a liquid-solid interface. Adv Mater 32(2): 1905696 (2020)

[109] Lin S Q, Xu L, Wang A C, Wang Z L. Quantifying electron-transfer in liquid-solid contact electrification and the formation of electric double-layer. Nat Commun 11: 399 (2020) 


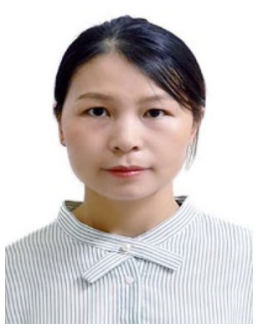

Linglin ZHOU. She received her Ph.D. degree from University of Science and Technology of China in 2018. Now she is a postdoctoral fellow in Beijing Institute of Nano-

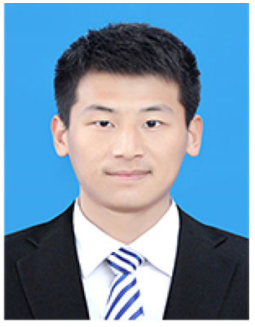

Di LIU. He received his B.S. degree in material science and engineering from Nanjing University of Aeronautics and Astronautics, China. Now he is a Ph.D. candidate

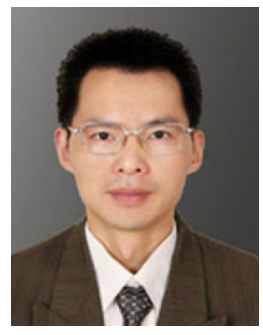

Jie WANG. He received his Ph.D. degree from $X_{i}$ 'an Jiaotong University in 2008, China. He is currently a professor at Beijing

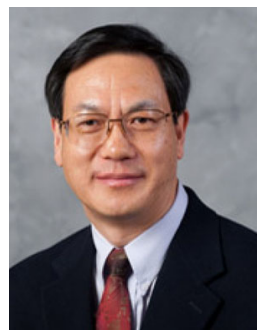

Zhong Lin WANG. He is the hightower chair in materials science and engineering and Regents' Professor at Georgia Tech, and founding director of the Beijing energy and Nanosystems, Chinese Academy of Sciences, China. Her current research focuses on the improvement of triboelectric nanogenerators and their applications in environmental pollutants treatment.

in Beijing Institute of Nanoenergy and Nanosystems, Chinese Academy of Sciences, China. His current research interest is triboelectric nanogenerators for energy harvesting and self-powered systems.

Institute of Nanoenergy and Nanosystems, Chinese Academy of Sciences, China. His current research interests focus on supercapacitors, nanogenerators, and self-powered system.

Institute of Nanoenergy and Nanosystems, China. Dr. Wang pioneered the field of nanogenerators and self-powered sensors, and coined piezotronics and piezo-phototronics for the third-generation semiconductors. 